\title{
Stationarity Tests for Irregularly Spaced ObSERVATIONS AND the EFfects of SAMPling FREQUENCY ON POWER*
}

\author{
Fabio Busetti \\ Research Department \\ Bank of Italy
}

\author{
A.M.Robert Taylor \\ Department of Economics \\ University of Birmingham
}

February 2004

\begin{abstract}
In this paper, starting from continuous-time local level unobserved components models for stock and flow data we derive locally best invariant (LBI) stationarity tests for data available at potentially irregularly spaced points in time. We demonstrate that the form of the LBI test differs between stock and flow variables. In cases where the data are observed at regular intervals throughout the sample we show that the LBI tests for stock and flow data both reduce to the form of the standard stationarity test in the discrete-time local level model. Here we also show that the asymptotic local power of the LBI test increases with the sampling frequency in the case of stock, but not flow, variables. Moreover, for a fixed time span we show that the LBI test for stock (flow) variables is (is not) consistent against a fixed alternative as the sampling frequency increases to infinity. We also consider the case of mixed frequency data in some detail, providing asymptotic critical values for the LBI tests for both stock and flow variables, together with a finite sample power study. Our results suggest that tests which ignore the infra-period aspect of the data involve rather small losses in efficiency relative to the LBI test in the case of flow variables, but can result in significant losses of efficiency when analysing stock variables.
\end{abstract}

Keywords: Stock and flow variables; local level model; locally best invariant test, sampling frequency, time aggregation.

Email: busetti.fabio@insedia.interbusiness.it, R.Taylor@bham.ac.uk

\footnotetext{
${ }^{*}$ We thank Marcus Chambers, Andrew Harvey, Rod McCrorie, Neil Shephard and seminar participants at Nuffield College, Oxford for their helpful comments and suggestions on earlier versions of this paper. We are especially indebted to Marcus Chambers who suggested the formulation of the observation equations used in our continuous-time unobserved components models for stock and flow variables. All errors are ours.
} 


\section{Introduction}

Consider the discrete-time local level, or random walk plus noise, unobserved components model of Harvey (1989,p.19), extended to allow for a deterministic kernel, $a_{t}$, for the univariate time series process $\left\{y_{t}\right\}$ :

$$
\begin{aligned}
& y_{t}=a_{t}+\mu_{t}+\varepsilon_{t}, \quad \varepsilon_{t} \sim \operatorname{NIID}\left(0, \sigma_{\varepsilon}^{2}\right) \\
& \mu_{t}=\mu_{t-1}+\eta_{t}, \quad \eta_{t} \sim N I I D\left(0, \sigma_{\eta}^{2}\right),
\end{aligned}
$$

$t=1, \ldots, T$. We assume the initial condition $\mu_{0}=0$, which implies no loss of generality, provided $a_{t}$ includes a constant and, for the present, that the irregular and level disturbance processes $\left\{\varepsilon_{t}\right\}$ and $\left\{\eta_{t}\right\}$ are uncorrelated, both temporally and contemporaneously.

In the context of (1.1)-(1.2) considerable theoretical and empirical interest has focused on the, so-called, stationarity testing problem. That is, defining the signal-to-noise ratio in (1.1)-(1.2) as $q=\sigma_{\eta}^{2} / \sigma_{\varepsilon}^{2}$, testing the null hypothesis of stationarity, $H_{0}: q=0$, against the unit root alternative, $H_{1}: q>0$. Setting $a_{t}=a$, a constant, Nyblom and Mäkeläinen (1983) [NM] demonstrate that the locally best invariant [LBI] test of $H_{0}$ against $H_{1}$ rejects for large values of the statistic

$$
\mathcal{N M}=\frac{\mathbf{e}^{\prime} \mathbf{W e}}{T \mathbf{e}^{\prime} \mathbf{e}}
$$

where $\mathbf{e}=\left(e_{1}, \ldots, e_{T}\right)^{\prime}$ is the $T$-vector of Ordinary Least Squares (OLS) residuals obtained from regressing $y_{t}$ on an intercept, $t=1, \ldots, T$, and $\mathbf{W}$ is the so-called random walk generating matrix with $(i, j)$ th element equal to the minimum of $i$ and $j, i, j=1, \ldots, T$. King and Hillier (1985) show that this is also a one-sided Lagrange multiplier test of $H_{0}$ against $H_{1}$. Nyblom (1986) has extended the analysis of NM to the case where $a_{t}=a+b t$, a linear time trend, in which case the LBI test is as given in (1.3) except that $\mathbf{e}$ is now the vector of OLS residuals from regressing $y_{t}$ on an intercept and trend. Further generalisations are provided by Nabeya and Tanaka (1988), Kwiatkowski et al. (1992), Busetti and Harvey (2001).

Tanaka (1996,p.368), inter alia, demonstrates that under the local alternative, $H_{c}: q=$ $c^{2} / T^{2}, c \geq 0$, as $T \rightarrow \infty$

$$
\mathcal{N} \mathcal{M} \Rightarrow \int_{0}^{1} V_{\xi}(r ; c)^{2} d r
$$

with

$$
V_{1}(r ; c) \equiv B_{1}(r)+c\left(\int_{0}^{r} W_{1}(s) d s-r \int_{0}^{1} W_{1}(\tau) d \tau\right)
$$




$$
V_{2}(r ; c) \equiv V_{1}(r ; c)-6 r(1-r) \int_{0}^{1} V_{1}(s ; c) d s,
$$

where " $\Rightarrow$ " denotes weak convergence of the associated probability measures, and the nomenclature $\xi=1,2$, is used exclusively throughout this paper to indicate whether the deterministic kernel is a constant, in which case $\xi=1$, or a linear time trend, in which case $\xi=2$. In (1.4), $B_{1}(r) \equiv W_{0}(r)-r W_{0}(1), r \in[0,1]$, is a standard Brownian bridge process, defined via the standard Brownian motion $W_{0}(r)$, while $W_{1}(r)$ is a standard Brownian motion process independent of $W_{0}(r){ }^{1}$

Where $c=0, H_{c}$ reduces to $H_{0}$ and hence (1.4) will give the limiting null distribution of the LBI statistic, $\mathcal{N M}$ of (1.3). For $\xi=1$ and $\xi=2$ these are respectively first- and second-level Cramér-von Mises distributions with one degree of freedom; see Harvey (2001) for further discussion on the Cramér-von Mises family of distributions. In what follows we will denote these Cramér-von Mises distributions by $V M_{\xi}(1), \xi=1,2$, upper tail critical values from which are provided in, for example, Kwiatkowski et al. (1992, p.166) [KPSS] and Harvey (2001, p.4,8). Moreover, from (1.4) the limiting (local) power function of $\mathcal{N} \mathcal{M}$ under $H_{c}$ for an $\alpha$-level test will be given by

$$
\pi_{\xi}(c) \equiv \operatorname{Pr}\left\{\int_{0}^{1} V_{\xi}(r ; c)^{2} d r>k_{\alpha, \xi}\right\}, \quad \xi=1,2,
$$

where $k_{\alpha, \xi}$ is such that $\operatorname{Pr}\left\{\int_{0}^{1} V_{\xi}(r ; 0)^{2} d r>k_{\alpha, \xi}\right\}=\alpha, \xi=1,2$. For both $\xi=1$ and $\xi=2$, (1.6) is monotonically increasing in $c$. These limiting power functions are graphed in Tanaka (1996, Figure 10.4, p.389). Under the fixed alternative $H_{1}: q>0, \mathcal{N} \mathcal{M}$ is of $O_{p}(T)$ and positive; see, inter alia, KPSS pp.165-9. Consequently, the LBI test is consistent against fixed alternatives.

Although the foregoing results were derived under the assumption of uncorrelated disturbances, Bailey and Taylor (2002) have recently demonstrated that the form of the LBI test, for a given deterministic kernel, is unaltered by allowing for contemporaneous correlation between $\varepsilon_{t}$ and $\eta_{t}$ : the, so-called, non-orthogonal model. Moreover, they demonstrate that the limiting distribution theory in (1.4) for $\mathcal{N} \mathcal{M}$ of (1.3) also remains appropriate under the non-orthogonal model.

The stationarity testing literature to date has started from the basic presumption that the data is generated by the regularly-spaced discrete-time local level model, (1.1)-(1.2).

\footnotetext{
${ }^{1}$ The result in (1.4) and those which follow also hold under considerably weaker, martingale difference, conditions on the irregular and level disturbance processes; see Stock (1994,pp.2745,2794-2799) for details.
} 
However, as argued in Harvey (1989,p.479), "A continuous time model is, in some ways, more fundamental than a discrete time model ... a good deal of the theory in economics and other subjects is based on continuous time models." Notably, continuous-time models provide a framework which allows us to handle irregularly-spaced data and highlights the important distinctions that exist, in particular when the data are irregularly-spaced, between stock and flow variables. Stocks are variables such as prices, unemployment, temperature, interest rates, and the capital stock which can, in principle, be observed at any given point in time, while flows are variables such as rainfall, income, and consumption expenditures which are defined with respect to an interval of time. It is perhaps surprising, therefore, that stationarity tests have been widely applied to both macroeconomic and financial data without any formal investigation into what effects the stock-flow distinction has on the underlying testing problem. Using continuous-time formulations of the local level model for stock and flow variables, we undertake a detailed exploration of these issues in this paper.

In section 2 we set out the continuous-time formulation of the local level model for both stock and flow variables and derive the corresponding discrete-time analogue models. In neither case do these analogue models reduce to the discrete-time model (1.1)-(1.2), except where the observations are regularly-spaced. Even then, in the case of flow variables one obtains the non-orthogonal discrete-time local level model. This then allows us, in section 3 , to derive the LBI tests for the null hypothesis that the signal-to-noise ratio in the continuous time model is zero against the alternative that it is positive in cases where the sample observations are irregularly spaced through time. We demonstrate that the form of the resulting LBI test, for a given pattern of intra-observation intervals, is different for stock vis-à-vis flow variables. The limiting null distributions of the LBI statistics are, in general, non-pivotal. An exception occurs where the data are regularly sampled. Here the LBI tests for stock and flow data both have pivotal Cramér-von Mises distributions.

In section 4 we analyse the case where the data are regularly sampled and show that for stock variables the local limiting power of the LBI test increases with the sampling frequency; that is, ceteris paribus, power is higher for $N$ years of monthly data than for $N$ years of annual data. Moreover, for stock variables we also show that the LBI test is consistent against fixed alternatives for cases where the continuous-time model is generated for a fixed time span but with the sampling interval tending to zero. In contrast, we show that for flow variables the local limiting power of the LBI test does not increase with the sampling frequency, and that 
the LBI test is not consistent when the time span is fixed and the sampling interval tends to zero. Interestingly, these findings contrast with what has been found for the Dickey-Fuller test by Phillips (1987), Perron (1991), Ng (1995) and Chambers (2004). However, this is perhaps not surprising given that our model effectively reverses the role of the null and the alternative hypotheses, relative to the Dickey-Fuller set-up. Loosely speaking, in both models a stock variable which admits a unit root appears "more nonstationary" when it is observed with higher frequency, while a parallel, but reversed, argument holds in the case of flow variables.

An interesting case of irregularly-spaced data is provided by mixed frequency data. An example of this is where some sub-sample of the complete data-set is observed annually and the remaining sub-sample comprises quarterly observations. In section 5 we derive explicit expressions for the LBI stationarity tests for mixed frequency data, as special cases of the tests derived in section 3 . We provide representations for the limiting null distributions of the LBI statistics, demonstrating that these depend on the fraction of the data observed in each sub-sample and on the relative observation frequencies in the two sub-samples. These representations differ considerably between stock and flow variables. A selection of critical values from these asymptotic distributions is provided. We also consider tests which are based on statistics modified, either by considering the two sub-samples separately or by data-aggregation, so as to have limiting null distributions belonging to the Cramér-von Mises family. The finite-sample power properties of these tests are compared, via Monte Carlo simulation, with the LBI tests. Our results suggest that the use of the aggregate data involves only a negligible losses in efficiency for the case of flow variables, but not for stock variables; in the latter case our simulations are decisively in favour of the exact LBI tests developed in this paper.

\section{Continuous-time local level models for stock and flow data and their discrete-time analogues}

The continuous-time analogue of the discrete-time random walk transition equation (1.2) is given by

$$
d \mu(t)=\xi_{2}(d t), \quad t>0
$$


where $\xi_{2}(d t)$ is a random measure, defined formally below. Formulating the continuous-time analogue of the observation equation (1.1) is more involved. Due to the static nature of (1.1), different continuous-time formulations of the observation equation will necessarily be required for the separate cases of stock and flow data, since the former are measured at single points in time while the latter are measured as integrals over the sampling interval. Consider first the case of stock data. Here the appropriate observation equation is given by

$$
y(t)=a(t)+\mu(t)+\varepsilon(t), \quad t>0
$$

where $a(t)$ is a deterministic kernel and $\varepsilon(t)$ is heuristically a serially uncorrelated meanzero process with variance $\sigma_{\varepsilon}^{2}$, as in Wymer (1993). For the case of flow variables, the corresponding observation equation is given by

$$
y(t) d t=[a(t)+\mu(t)] d t+\xi_{1}(d t), \quad t>0
$$

where $\xi=\left(\xi_{1}, \xi_{2}\right)^{\prime}$ is a two-dimensional random measure, defined on all subsets of the halfline $0<t<\infty$ with finite Lebesgue measure, satisfying Assumption 2.1 of Bergstrom (1986), with $E[\xi(d t)]=0$ and $E\left[\xi(d t) \xi^{\prime}(d t)\right]=(d t) \Sigma, \quad \Sigma=\operatorname{diag}\left(\sigma_{\varepsilon}^{2}, \sigma_{\eta}^{2}\right)$. Heuristically speaking, $\xi(d t)$ can be thought of as a continuous-time white noise process (see Bergstrom, 1984,p.1157) for further discussion, and, moreover, (2.3) may be viewed as (2.2) multiplied through by $d t$ and with $\varepsilon(t) d t$ replaced by $\xi_{1}(d t)$.

Our continuous-time local level models for stock and flow data are therefore given by (2.2)(2.1) and (2.3)-(2.1), respectively. As with (1.1)-(1.2), for both (2.2)-(2.1) and (2.3)-(2.1) we may assume the initial condition $\mu(0)=0$ with no loss of generality, provided $a(t)$ contains an intercept term. Harvey and Stock (1993,pp.58-59) and Chambers and McGarry (2002) specify the discrete-time observation equations for stock and flow data directly. However, a comparison of (2.6)-(2.7) and (2.9)-(2.10), below, with Equations (9),(10),(12),(25) of Harvey and Stock (1993,pp.58-64) shows that the discrete-time analogues of (2.2)-(2.1) and (2.3)(2.1) coincide with the discrete-time models specified for stock and flow variables in Harvey and Stock (1993). This point is also recognised by Chambers and McGarry (2002) who argue in the context of the discrete-time analogues that “... the random disturbance ... can be interpreted either as the discrete time realization of a genuine irregular component in continuous time .. or as a measurement error that is associated with the discrete time sampling of the continuous time process", op. cit., p.393. 
Our formulations of the local level model for stock and flow variables provide a general framework from which to examine the corresponding exact discrete-time models, where the observations are sampled at possibly irregular intervals. As with the stationarity tests outlined for the case of the discrete-time local level model, (1.1)-(1.2), in section 1, our interest focuses on testing the null hypothesis of stationarity against the unit root alternative in the continuous-time local level models. That is, we wish to test the following hypothesis regarding the structural, signal-to-noise ratio, parameter $^{2} q=\sigma_{\eta}^{2} / \sigma_{\varepsilon}^{2}$

$$
H_{0}: q=0
$$

versus

$$
H_{1}: q>0
$$

in (2.2)-(2.1)for stock variables and in (2.3)-(2.1) for flow variables, using observations on the process which are available at possibly irregular spaced discrete time points. In particular, we suppose that the data are observed over the interval $0<t \leq N$, where $N$ denotes the span of the data, at $T$ irregular observation times $\left\{t_{\tau}\right\}, \tau=1, \ldots, T$, where $t_{\tau}=t_{\tau-1}+\delta_{\tau}$, $t_{0}=0$, with the intra-observation intervals, $\delta_{\tau}$, expressed in calendar time units, such that $\sum_{\tau=1}^{T} \delta_{\tau}=N$.

In a series of influential papers Bergstrom (1983,1984 and 1985), inter alia, sets out the theoretical treatment of continuous-time autoregressive models and their estimation by Choleski factorization of the Gaussian likelihood. Building upon this work, Harvey (1989, Ch.9) and Harvey and Stock (1993) consider a general class of state space models in continuous-time where the Gaussian likelihood estimation is carried out via the Kalman filter. The continuous-time models in (2.2)-(2.1) and (2.3)-(2.1) may be regarded as simple examples from this class of state space models. ${ }^{3}$ They are, nevertheless, important from the point of view of constructing stationarity tests for the case of irregularly spaced observations on stock and flow data respectively. We now derive the discrete-time analogues of (2.2)-(2.1) and $(2.3)-(2.1)$.

\footnotetext{
${ }^{2}$ Notice that the signal-to-noise ratios which are subsequently obtained for the discrete-time analogues for stock and flow variables are derived, rather than structural, parameters.

${ }^{3}$ More sophisticated models, including those with common trends and co-integration in continuous time, have been considered by Harvey and Stock (1988, 1989), Phillips (1991) and Comte (1999), inter alia.
} 


\subsection{Stock Variables}

Consider first the case of a stock variable. Let $y_{\tau}=y\left(t_{\tau}\right), \mu_{\tau}=\mu\left(t_{\tau}\right), a_{\tau}=a\left(t_{\tau}\right)$, and $\varepsilon_{\tau}=\varepsilon\left(t_{\tau}\right)$. Evaluating (2.2)-(2.1) at $t=t_{\tau}$, yields the discrete-time analogue

$$
\begin{aligned}
& y_{\tau}=a_{\tau}+\mu_{\tau}+\varepsilon_{\tau}, \\
& \mu_{\tau}=\mu_{\tau-1}+\eta_{\tau}, \quad \mu_{0}=0
\end{aligned}
$$

where $\varepsilon_{\tau}$ and $\eta_{\tau}$ are mean zero, serially uncorrelated and mutually orthogonal disturbances with variances $\sigma_{\varepsilon}^{2}$ and $\delta_{\tau} \sigma_{\eta}^{2}$ respectively. It is important to notice that, unlike the discretetime model in (1.1)-(1.2), the disturbances driving the level equation, (2.7), are heteroscedastic when the observations are irregularly sampled; that is, where $\delta_{\tau}$ is not constant through the observed sample. In the case where $a(t)=a$, then so $a_{\tau}=a$, so that a constant in the continuous-time local-level model, (2.2)-(2.1), implies a constant in the discrete-time analogue, (2.6)-(2.7). However, if $a(t)=a+b t$, a linear trend, then $a_{\tau}=a+b t_{\tau}$ which will not, in general, be of the form of a linear trend in $\tau$. An obvious exception occurs where $\delta_{\tau}$ is fixed through the sample (the observations occur at regular time points). In this case (2.6)-(2.7) clearly reduces to a discrete-time local level of the form (1.1)-(1.2).

\subsection{Flow Variables}

An observed flow variable, which we denote by $Y_{\tau}$, is obtained by integrating the (unobserved) continuous-time analogue $y(t)$ over the interval $\left(t_{\tau-1}, t_{\tau}\right]$; viz.,

$$
Y_{\tau}=\int_{t_{\tau-1}}^{t_{\tau}} y(t) d t
$$

As demonstrated in Appendix A, one therefore obtains that the discrete-time analogue of (2.3)-(2.1) is given by

$$
\begin{aligned}
& Y_{\tau}=A_{\tau}+\delta_{\tau} \mu_{\tau}+\varepsilon_{\tau}^{*}, \\
& \mu_{\tau}=\mu_{\tau-1}+\eta_{\tau}, \quad \mu_{0}=0
\end{aligned}
$$

where $A_{\tau}=\int_{t_{\tau-1}}^{t_{\tau}} a(t) d t, \mu_{\tau}=\mu\left(t_{\tau}\right)$ and $\varepsilon_{\tau}^{*}$ and $\eta_{\tau}$ are mean zero, serially uncorrelated disturbances. However, and in contrast to the case of a stock variable, the disturbance in the measurement equation $\varepsilon_{\tau}^{*}$ is contemporaneously (but not temporally) correlated with 
that of the transition equation $\eta_{\tau}$, viz.,

$$
\operatorname{Var}\left(\begin{array}{l}
\varepsilon_{\tau}^{*} \\
\eta_{\tau}
\end{array}\right)=\left(\begin{array}{ll}
\delta_{\tau}^{3} \sigma_{\eta}^{2} / 3+\delta_{\tau} \sigma_{\varepsilon}^{2} & -\frac{1}{2} \delta_{\tau}^{2} \sigma_{\eta}^{2} \\
-\frac{1}{2} \delta_{\tau}^{2} \sigma_{\eta}^{2} & \delta_{\tau} \sigma_{\eta}^{2}
\end{array}\right)
$$

see also Harvey and Stock (1993). ${ }^{4}$

As regards the deterministic kernel, if $a(t)=a$, then $A_{\tau}=a \delta_{\tau}$, so that a constant in the continuous-time local level model (2.3)-(2.1) does not imply a constant in the discrete-time analogue model, unless $\delta_{\tau}$ is fixed through the sample. Moreover, if $a(t)=a+b t$, a linear trend, then $A_{\tau}=\left(a+b t_{\tau}-\frac{b}{2} \delta_{\tau}\right) \delta_{\tau}$ : as with the case of a stock variable, this reduces to a standard linear trend where $\delta_{\tau}$ is constant across the sample.

It is crucial to notice that, and again in contrast to the case of stock variables, even where $\delta_{\tau}$ is constant across the sample, (2.9)-(2.10) does not reduce to the class of discrete-time local level models as in (1.1)-(1.2), due to the contemporaneous correlation between $\varepsilon_{\tau}^{*}$ and $\eta_{\tau}$. However, since our interest in this paper lies solely in developing optimal tests of $H_{0}$ of (2.4) against $H_{1}$ of (2.5), we know from the results of Bailey and Taylor (2002) that, in this regard, the orthogonal and non-orthogonal local level models may be treated as if they were the same. This point is pursued further in section 3.3 .

\section{The LBI Test against the Presence of a Random Walk Component}

In this section we derive the LBI test of $H_{0}$ of (2.4) against $H_{1}$ of (2.5) for the cases of both stock and flow variables observed at (possibly irregularly spaced) discrete points. These results show that there are significant and important differences between the two cases. The LBI tests are obtained using the framework of King and Hillier (1985).

The structure of the resulting LBI test statistics is shown to depend on the intraobservation intervals, $\delta_{\tau}, \tau=1, \ldots, T$, which may be regular or irregular. The former case is

\footnotetext{
${ }^{4}$ Notice that Harvey and Stock (1993) write $(2.9)$ with $\mu_{\tau-1}$ in place of $\mu_{\tau}$, and redefine $\varepsilon_{\tau}^{*}$ accordingly. Save for the minus sign in the expression for $\operatorname{Cov}\left(\varepsilon_{\tau}^{*}, \eta_{\tau}\right)$, the two representations coincide. Moreover, Chambers and McGarry (2002,p.395) choose a different formulation where the disturbance terms in the discrete-time observation and transition equations are uncorrelated (both temporally and contemporaneosuly) but the latter follows an MA(1) process. The non-orthogonal representation, (2.9)-(2.10), is clearly the more useful in the context of this paper.
} 
pursued further in sections 3.3 and 4 , where it is shown that the statistics simplify considerably. Two interesting examples of the latter are the mixed frequency data and missing data cases. The first example is pursued in detail in section 5. Stationarity tests in the presence of missing data obtain as special cases of the LBI tests outlined for stock and flow variables in sections 3.1 and 3.2 respectively, and we shall not pursue such tests in any further detail here. However, for a set-up which is analogous to our stock variable case and under certain restrictions on the intra-observation intervals, Nishino (2002) provides an exploration of stationarity testing with missing data.

\subsection{Stock Variables}

Let $\mathbf{y}_{S}=\left(y_{1}, \ldots, y_{T}\right)^{\prime}$ denote the $T \times 1$ vector of observations when the observed variable is a stock. From (2.6)-(2.7) and assuming Gaussianity, we immediately obtain that

$$
\mathbf{y}_{S} \sim N\left(\mathbf{a}_{S}, \sigma_{\varepsilon}^{2} \mathbf{\Omega}_{S}(q)\right)
$$

where $\mathbf{a}_{S}$ is a $T \times 1$ vector with $\tau$-th element $a_{\tau}$, and $\boldsymbol{\Omega}_{S}(q)=\mathbf{I}_{T}+q \mathbf{V}_{\delta}$, with $\mathbf{V}_{\delta}$ a $(T \times T)$ matrix with $(i, j)$ th element equal to the minimum of $t_{i}$ and $t_{j}, i, j=1, \ldots, T$.

A straightforward application of Equation 6 of King and Hillier (1985,p.99) then yields that the LBI test of $H_{0}$ of (2.4) against $H_{1}$ of (2.5) in the case of a stock variable rejects for large values of the statistic

$$
L_{S}=\frac{\mathbf{e}_{S}^{\prime} \mathbf{V}_{\delta} \mathbf{e}_{S}}{T \mathbf{e}_{S}^{\prime} \mathbf{e}_{S}} .
$$

In (3.2), $\mathbf{e}_{S}=\left(e_{1, S}, \ldots, e_{T, S}\right)^{\prime}$ is the vector of OLS residuals obtained from the projection onto the deterministic component $\mathbf{a}_{S}$. For the linear trend case, $a(t)=a+b t, \mathbf{e}_{S}$ is obtained from regressing $y_{\tau}$ on $z_{S, \tau}=\left(1, t_{\tau}\right)^{\prime}$, while in the case of a constant level, $a(t)=a$, the elements of $\mathbf{e}_{S}$ are simply the deviations from the sample mean.

The numerator of the statistic $L_{S}$ of (3.2) may be written equivalently as the weighted sum of squared (reverse) partial sums of the OLS residuals,

$$
\mathbf{e}_{S}^{\prime} \mathbf{V}_{\delta} \mathbf{e}_{S}=\sum_{\tau=1}^{T} \delta_{\tau}\left(\sum_{j=\tau}^{T} e_{j, S}\right)^{2}
$$

the weights being the sequence of intra-observation intervals $\left\{\delta_{1}, \ldots, \delta_{T}\right\}$. Because of this dependence on the $\delta_{1}, \ldots, \delta_{T}$ the null distribution of $L_{S}$ cannot be easily obtained. An obvious exception, considered in the section 3.3, occurs when the observations are sampled at 
regularly spaced intervals; that is, where $\delta_{\tau}$ is constant across the sample. However, since $L_{S}$ is formed as the ratio of two quadratic forms in Gaussian variables, the exact distribution of $L_{S}$ under both $H_{0}$ of $(2.4)$ and $H_{1}$ of $(2.5)$, for a given sequence, $\left\{\delta_{1}, \ldots, \delta_{T}\right\}$, can be obtained using the well-known Imhof (1961) routine.

\subsection{Flow Variables}

Consider now the case of a flow variable. Let $\mathbf{y}_{F}=\left(Y_{1}, \ldots, Y_{T}\right)^{\prime}$ denote the $T \times 1$ vector of observations on the flow variable and let $\mathbf{D}=\operatorname{diag}\left(\delta_{1}, \ldots, \delta_{T}\right)$. In Appendix $\mathrm{B}$ it is demonstrated that, from (2.9)-(2.10) and assuming Gaussianity,

$$
\mathbf{y}_{F} \sim N\left(\mathbf{a}_{F}, \sigma_{\varepsilon}^{2} \mathbf{D}^{\frac{1}{2}} \boldsymbol{\Omega}_{F}(q) \mathbf{D}^{\frac{1}{2}}\right),
$$

where $\mathbf{a}_{F}$ is a $T \times 1$ vector with $\tau$-th element $A_{\tau}$, and

$$
\boldsymbol{\Omega}_{F}(q)=\mathbf{I}_{T}+q \mathbf{D}^{\frac{1}{2}} \mathbf{V}_{\delta} \mathbf{D}^{\frac{1}{2}}+q \mathbf{V}_{*},
$$

with $\mathbf{V}_{\delta}$ defined as above, and where $\mathbf{V}_{*}$ is a symmetric matrix with $(i, j)$ th element, $i \leq$ $j=1, \ldots, T$,

$$
\left[\mathbf{V}_{*}\right]_{i j}= \begin{cases}-\frac{2}{3} \delta_{i}^{2} & i=j \\ -\frac{1}{2} \delta_{i}^{\frac{3}{2}} \delta_{j}^{\frac{1}{2}} & i<j\end{cases}
$$

Again using Equation 6 of King and Hillier (1985,p.99), it then follows that the LBI test of $H_{0}$ of (2.4) against $H_{1}$ of (2.5) for the case of a flow variable rejects for large values of the statistic

$$
L_{F}=\frac{\mathbf{e}_{F}^{\prime}\left(\mathbf{D}^{\frac{1}{2}} \mathbf{V}_{\delta} \mathbf{D}^{\frac{1}{2}}+\mathbf{V}_{*}\right) \mathbf{e}_{F}}{T \mathbf{e}_{F}^{\prime} \mathbf{e}_{F}} .
$$

In the context of (3.6), $\mathbf{e}_{F}=\left(e_{1, F}, \ldots, e_{T, F}\right)^{\prime}$ is the vector of OLS residuals from regressing $\mathbf{D}^{-\frac{1}{2}} \mathbf{y}_{F}$ on the deterministic component $\mathbf{D}^{-\frac{1}{2}} \mathbf{a}_{F}$. For example, in the case of a constant level $a(t)=a$, so that $A_{\tau}=a \delta_{\tau}$, one obtains that $e_{\tau, F}=\delta_{\tau}^{-\frac{1}{2}}\left(Y_{\tau}-\delta_{\tau} \sum Y_{\tau} / N\right), \tau=1, \ldots, T$.

Noting that the variance of $\mathbf{e}_{F}^{\prime} \mathbf{V}_{*} \mathbf{e}_{F}$ is of smaller order (in $T$ ) than that of $\mathbf{e}_{F}^{\prime} \mathbf{D}^{\frac{1}{2}} \mathbf{V}_{\delta} \mathbf{D}^{\frac{1}{2}} \mathbf{e}_{F}$ under both $H_{0}$ of (2.4) and $H_{1}$ of (2.5), it follows that $L_{F}$ is asymptotically equivalent to the simplified statistic

$$
L_{F}^{*}=\frac{\mathbf{e}_{F}^{\prime} \mathbf{D}^{\frac{1}{2}} \mathbf{V}_{\delta} \mathbf{D}^{\frac{1}{2}} \mathbf{e}_{F}}{T \mathbf{e}_{F}^{\prime} \mathbf{e}_{F}} .
$$

In what follows we will therefore concentrate our attention on $L_{F}^{*}$. As with $L_{S}$ of $(3.2)$, the numerator of $L_{F}^{*}$ of $(3.7)$ depends on the sequence of intra-observation intervals $\left\{\delta_{1}, \ldots, \delta_{T}\right\}$. 
For a given sequence, $\left\{\delta_{1}, \ldots, \delta_{T}\right\}$, the exact distribution of $L_{F}^{*}$, and indeed $L_{F}$, can again be obtained using the Imhof (1961) routine, while matters again simplify greatly when $\delta_{\tau}$ is constant across the sample.

\subsection{LBI Tests when $\delta_{\tau}$ is Constant}

Suppose now that the intra-observation interval $\delta_{\tau}$ is constant, taking the fixed value $\delta$, known as the sampling interval, throughout the sample. As a consequence we obtain that, $\mathbf{V}_{\delta}=\delta \mathbf{W}$, where $\mathbf{W}$ is the random-walk generating matrix introduced in section 1.

Consider first the case of a stock variable. Here we immediately obtain from (3.2) that

$$
L_{S}=\frac{\delta \mathbf{e}_{S}^{\prime} \mathbf{W} \mathbf{e}_{S}}{T \mathbf{e}_{S}^{\prime} \mathbf{e}_{S}}
$$

where $\mathbf{e}_{S}$ are as defined below (3.2) but with $\delta_{\tau} \equiv \delta$. This implies that the elements of $\mathbf{e}_{S}$ are simply the de-meaned or de-trended observations on $y_{t}$ for the case of a constant or linear trend respectively. Similarly, in the case of flow variables, we obtain from (3.7) that

$$
L_{F}^{*}=\frac{\delta^{2} \mathbf{e}_{F}^{\prime} \mathbf{W} \mathbf{e}_{F}}{T \mathbf{e}_{F}^{\prime} \mathbf{e}_{F}}
$$

where $\mathbf{e}_{F}$ are similarly the de-meaned or de-trended observations on the flow variable $Y_{\tau}$ for a constant or linear trend respectively.

Given the distributional properties established for the stock data, $y_{t}$, in (3.1) and the flow data, $Y_{\tau}$, in (3.4), it follows immediately from (1.4) and applications of the continuous mapping theorem [CMT] that, under $H_{0}$ of $(2.4), L_{S}$ and $L_{F}^{*}$ weakly converge, as $T \rightarrow \infty$, to $\delta$ times a $V M_{\xi}(1)$ distribution and $\delta^{2}$ times a $V M_{\xi}(1)$ distribution, respectively. ${ }^{5}$ Moreover, notice from the discussion immediately following (3.6), that this result will also hold for $L_{F}$. Indeed, we can say rather more than that in this case: it is straightforward to show that when $\delta_{\tau}$ is constant throughout the sample, for a given value of $T,\left(\mathbf{e}_{F}^{\prime} \mathbf{V}_{*} \mathbf{e}_{F}\right) /\left(T \mathbf{e}_{F}^{\prime} \mathbf{e}_{F}\right)$ collapses to a constant with respect to $\mathbf{e}_{F}$. Consequently, $L_{F}$ and $L_{F}^{*}$ will have identical critical regions. There is, therefore, no loss in efficiency from considering the test based on the $L_{F}^{*}$ statistic in this case, which is exact LBI.

In practice, when constructing the statistics $L_{S}$ and $L_{F}^{*}$ we may set the calendar time unit $\delta_{\tau}=1$ without altering the critical regions of the associated tests. We may therefore define

\footnotetext{
${ }^{5}$ Recall from section 1 that the nomenclature $V M_{\xi}(1), \xi=1,2$, denotes respectively first- and second-level Cramér-von Mises distributions with one degree of freedom.
} 
the resulting LBI statistics as $\bar{L}_{S}(\delta) \equiv L_{S} / \delta$ and $\bar{L}_{F}^{*}(\delta) \equiv L_{F}^{*} / \delta^{2}$, respectively, which clearly coincide with the standard discrete-time stationarity test statistic, $\mathcal{N} \mathcal{M}$ of (1.3), applied to the observed stock and flow data, respectively. Moreover, both $\bar{L}_{S}(\delta)$ and $\bar{L}_{F}^{*}(\delta)$ will clearly have pivotal $V M_{\xi}(1)$ limiting null distributions so that the critical values tabulated in KPSS and Harvey (2001) may be applied directly. For the remainder of this paper, where $\delta_{\tau}$ is fixed throughout the sample, any reference to LBI tests will be taken to mean those based on the statistics $\bar{L}_{S}(\delta)$ and $\bar{L}_{F}^{*}(\delta)$.

Empirical applications of the standard stationarity tests of section 1 have concentrated in the main on regularly spaced macroeconomic aggregates, such as income, output and consumption, which are, by definition, flow variables and, hence, will generate non-orthogonal discrete-time local level models; see the discussion at the end of section 2. Although Bailey and Taylor (2002) motivated their research from purely statistical grounds, our results demonstrate that their findings are also of central importance to the problem of stationarity testing in economic data. They tell us that the standard stationarity test will be exact LBI and with the same limiting null distribution, irrespective of whether the data is generated as a stock or flow variable, provided the data are observed at regularly spaced intervals. However, as we shall see in the next section, the power properties of the LBI tests do differ across the stock and flow cases. Specifically, we show that the local limiting power function of the LBI test for stock (flow) data depends (does not depend) on the sampling frequency, $\delta^{-1}$, and that for a fixed data span the LBI test for stock (flow) data is consistent (not consistent) against fixed alternatives as the sampling frequency tends to infinity.

\section{Test Power and the Sampling Frequency}

In this section we suppose that the underlying process is generated by the continuous-time local level model (2.2)-(2.1) for stock variables or (2.3)-(2.1) for flow variables, and that observations on $y(t)$ are made at intervals of length $\delta>0$ over the interval $0<t \leq N$, where $N$ denotes the span of the data. The number of observations is therefore given by $T=N / \delta$, and this quantity will clearly increase if either $N$ increases or $\delta$ decreases, or both. Our aim in this section is to study the large sample power properties of the LBI tests appropriate for stock and flow variables. We first analyse in section 4.1 the behaviour of the test statistics under a sequence of local alternatives on the signal-to-noise ratio in the underlying continuous 
time models, which are useful for computing the local limiting power function of the tests as the time span increases to infinity. Subsequently, in section 4.2 we look at the limiting behaviour of the statistics under fixed alternatives in order to establish the consistency (or otherwise) of the tests. We show that fundamentally different results emerge for stock and flow variables. Monte Carlo simulation results and two empirical illustrations are provided in section 4.3 and 4.4 respectively.

\subsection{Behaviour under Local Alternatives}

The structural parameter of interest in both (2.2)-(2.1) and (2.3)-(2.1), is the signal-to-noise ratio, $q=\sigma_{\eta}^{2} / \sigma_{\varepsilon}^{2}$. For this parameter, a sequence of alternatives local to $H_{0}$ of $(2.4)$ in the span of the data, $N$, is therefore given by

$$
H_{N}(c): q_{N}=c^{2} / N^{2}, \quad c \geq 0
$$

The subscript $N$ has been placed on the signal-to-noise ratio to highlight that this corresponds to a local alternative hypothesis and also to distinguish it from the nomenclature used in section 1. Notice that $\sigma_{\eta}^{2}=c^{2} \sigma_{\varepsilon}^{2} N^{-2}$, under (4.1). Moreover, for $c=0, H_{N}(c)$ again reduces to $H_{0}$.

We now analyse the power properties of the LBI tests for both stock and flow variables under $H_{N}(c)$ of (4.1) as $N \rightarrow \infty$. We show that for stock variables asymptotic local power is an increasing function of the sampling frequency $\delta^{-1}$, while for flow variables power is unaffected by $\delta^{-1}$. As is subsequently demonstrated numerically by Monte Carlo methods in section 4.3 , the asymptotic local distribution theory provides a good approximation to the finite sample impact of the sampling frequency on power in both cases.

Consider first the case of a stock variable. It was demonstrated in section 2 that the exact discrete time analogue of the continuous-time model (2.2)-(2.1) is a random walk plus noise model with variances $\sigma_{\varepsilon}^{2}$ and $\delta \sigma_{\eta}^{2}$ respectively, observed for $T=N / \delta$ periods. Using (2.6)-(2.7), the derived signal-to-noise ratio, say $q_{T, S}$ (where we use $S$ to denote stock), in the discrete-time analogue under $H_{N}(c)$ of $(4.1)$ is therefore given by

$$
q_{T, S}=\frac{\delta c^{2} \sigma_{\varepsilon}^{2}}{\sigma_{\varepsilon}^{2} N^{2}}=\frac{\delta c^{2}}{T^{2} \delta^{2}} \equiv \frac{c_{S}^{2}}{T^{2}},
$$

where $c_{S} \equiv c / \sqrt{\delta}$, which is clearly an increasing function of the sampling frequency, $\delta^{-1}$. 
Therefore, it immediately follows from the results in section 3.3 and a comparison with (1.4) that, for given values of $c$ and $\xi, \pi_{\xi}(c / \sqrt{\delta})$ will be an increasing function of the sampling frequency. The implication of this is that the asymptotic local power of the LBI test in the case of a stock variable will be higher the larger is the sampling frequency, ceteris paribus, being a function of $c / \sqrt{\delta}$.

Consider now the model for a flow variable. Noting again from Bailey and Taylor (2002) that the contemporaneous correlation between the signal and noise processes has no effect on the local limiting distribution of the LBI statistic, exactly the same line of argument can be used to analyse the power properties of the LBI test for flow variables.

Using (2.9)-(2.10), noting that in this case the signal is $\delta \mu_{\tau}$, it follows that the derived signal-to-noise ratio for a discretely sampled flow variable, say $q_{T, F}$ (where $F$ denotes flow), under the local alternative $H_{N}(c)$ is given by

$$
q_{T, F}=\frac{\delta^{3} c^{2} \sigma_{\varepsilon}^{2} / N^{2}}{\frac{1}{3} \delta^{3} c^{2} \sigma_{\varepsilon}^{2} / N^{2}+\delta \sigma_{\varepsilon}^{2}}=\frac{c^{2}}{\frac{1}{3} c^{2}+T^{2}} \equiv \frac{c_{F}^{2}}{T^{2}},
$$

where

$$
c_{F} \equiv \sqrt{\frac{c^{2}}{\frac{1}{3} \frac{c^{2}}{T^{2}}+1}}
$$

and is clearly such that $\lim _{T \rightarrow \infty} c_{F}=c$. As $N \rightarrow \infty$ with $\delta$ fixed, we see from (4.3) that the local limiting power of the LBI test in the case of flow data depends only on $\xi$ and the noncentrality parameter, $c$, of (4.1). Crucially, it does not depend on the sampling frequency $\delta^{-1}$. Consequently, and in contrast to the case of stock variables, the local limiting power of the LBI test for a given non-centrality parameter, $c$, will not increase with the sampling frequency.

\subsection{Behaviour under Fixed Alternatives}

We now consider the continuous time model for stock and flow variables (2.2)-(2.1) and (2.3)(2.1) under the hypothesis that the signal-to-noise ratio $q=\sigma_{\eta}^{2} / \sigma_{\varepsilon}^{2}$ is fixed and strictly greater than zero; that is, we are interested in the behaviour of the tests under a fixed alternative rather than under the local alternative (4.1) of the previous subsection. In particular we will show that, for a fixed time span $N$, as the sampling frequency tends to infinity $(\delta \rightarrow 0)$ the LBI test is consistent for the case of stock variables but it is not consistent for flows. Where 
$N \rightarrow \infty$ the test is consistent for both stock and flow data. The proof for this case is not provided here since it follows immediately from KPSS and Bailey and Taylor (2002).

Consider first the case of a stock variable. From (2.6)-(2.7), the discrete time analogue, observed at a fixed time interval $\delta$, can be written as

$$
\begin{aligned}
& y_{\tau}=a_{\tau}+\delta^{\frac{1}{2}} \mu_{\tau}^{*}+\varepsilon_{\tau}, \quad \tau=1,2, \ldots, T \equiv N / \delta \\
& \mu_{\tau}^{*}=\mu_{\tau-1}^{*}+\eta_{\tau}^{*}, \quad \mu_{0}^{*}=0
\end{aligned}
$$

where $\varepsilon_{\tau}, \eta_{\tau}^{*}$ are serially uncorrelated and mutually orthogonal Gaussian disturbances with variances $\sigma_{\varepsilon}^{2}$ and $\sigma_{\eta}^{2}$ respectively, and $a_{\tau}=X_{\tau}^{\prime} \beta$ is a deterministic component represented in terms of a $k$-dimensional non-stochastic regressor $X_{\tau}$ with associated coefficient vector $\beta$. We assume that $X_{\tau}$ satisfies standard regularity conditions as in Phillips and Xiao (1998); that is, there exists a scaling matrix $\Lambda_{T}$ and a bounded piecewise continuous function $X(r)$ such that: (a) $\Lambda_{T} X_{\lfloor T r\rfloor} \rightarrow X(r)$ as $T \rightarrow \infty$ uniformly in $r \in[0,1]$; and (b) $\int_{0}^{1} X(r) X(r)^{\prime} d r \rightarrow Q_{X}$, a positive definite matrix, as $T \rightarrow \infty$. These conditions are clearly met if the deterministic component is a constant level or a linear trend, but they also allow for more general functions such as piecewise polynomial trends.

From section 3.3, the LBI statistic takes the form

$$
\bar{L}_{S}(\delta)=\frac{T^{-2} \sum_{\tau=1}^{T}\left(\sum_{j=\tau}^{T} e_{j}\right)^{2}}{T^{-1} \sum_{\tau=1}^{T} e_{\tau}^{2}},
$$

where $e_{\tau}$ is the $\tau$-th element of the vector of OLS residuals $\mathbf{e}_{S}$, obtained from regressing the observation vector $\mathbf{y}_{S}=\left(y_{1}, \ldots, y_{T}\right)^{\prime}$ on the deterministic variables $\mathbf{X}_{S}=\left(X_{1}^{\prime}, \ldots, X_{T}\right)^{\prime}$. As discussed in section 3.3, (4.4) is the usual form of the LBI test of stationarity for data sampled at constant intervals.

For a $T$-dimensional vector $\mathbf{z}$ denote by $\overline{\mathbf{z}}$ its orthogonal projection onto the space spanned by $\mathbf{X}_{S}$, i.e. $\overline{\mathbf{z}}=\mathbf{X}_{S}\left(\mathbf{X}_{S}^{\prime} \mathbf{X}_{S}\right)^{-1} \mathbf{X}_{S}^{\prime} \mathbf{z}$, with $\bar{z}_{\tau}$ being the $\tau$-th element of $\overline{\mathbf{z}}$; clearly, if $X_{\tau}$ is a constant regressor then $\bar{z}_{\tau}$ is just the average of the elements of $\mathbf{z}$. Then we can write the OLS residuals as $e_{\tau}=y_{\tau}-\bar{y}_{\tau}=\delta^{\frac{1}{2}}\left(\mu_{\tau}^{*}-\bar{\mu}_{\tau}^{*}\right)+\left(\varepsilon_{\tau}-\bar{\varepsilon}_{\tau}\right)$. Now, as $\delta \rightarrow 0$ with $N$ fixed, and recalling that $T=N / \delta$, standard applications of the Invariance Principle and the Continuous Mapping Theorem then yield, for $r \in[0,1]$, the following limiting results,

$$
\begin{gathered}
T^{-\frac{1}{2}}\left(\mu_{[T r]}^{*}-\bar{\mu}_{[T r]}^{*}\right) \Rightarrow \sigma_{\eta}(W(r)-\bar{W}(r))=O_{p}(1), \\
T^{-\frac{3}{2}} \sum_{j=[T r]}^{T}\left(\mu_{[T r]}^{*}-\bar{\mu}_{[T r]}^{*}\right) \Rightarrow \sigma_{\eta} \int_{r}^{1}(W(r)-\bar{W}(r)) d r=O_{p}(1),
\end{gathered}
$$


where $W(r)$ is a standard Brownian motion process, $\bar{W}(r) \equiv X^{\prime}(r) Q_{X}^{-1} \int_{0}^{1} X^{\prime}(r) W(r) d r$ and $[\cdot]$ denotes the integer part of its argument. Notice that if $X_{\tau}$ is a constant regressor, then $\bar{W}(r) \equiv \int_{0}^{1} W(r) d r$.

Therefore we immediately obtain from (4.5) and (4.6), that $\left(\mu_{[T r]}^{*}-\bar{\mu}_{[T r]}^{*}\right)=O_{p}\left(\delta^{-\frac{1}{2}}\right)$ and $\sum_{j=[T r]}^{T}\left(\mu_{[T r]}^{*}-\bar{\mu}_{[T r]}^{*}\right)=O_{p}\left(\delta^{-\frac{3}{2}}\right)$, while using similar arguments $\left(\varepsilon_{[T r]}-\bar{\varepsilon}_{[T r]}\right)=O_{p}(1)$ and $\sum_{j=[T r]}^{T}\left(\varepsilon_{[T r]}-\bar{\varepsilon}_{[T r]}\right)=O_{p}\left(\delta^{-\frac{1}{2}}\right)$. The numerator of the LBI statistic (4.4) is then seen to be of $O_{p}\left(\delta^{-1}\right)$ while, since $e_{[T r]}=O_{p}(1)$, the denominator is of $O_{p}(1)$. Consequently, as $\delta \rightarrow 0$ with fixed time span $N$, the LBI statistic (4.4) is of $O_{p}\left(\delta^{-1}\right)$ and the consistency property of the test follows. The qualitative prediction of this large sample result also appears evident in the finite-sample simulation results reported in Table 1 of section 4.3.

Consider now the case of a flow variable. From (2.9)-(2.10) the discrete time analogue, observed at a fixed time interval $\delta$, can be written as

$$
\begin{aligned}
& Y_{\tau}=A_{\tau}+\delta^{\frac{3}{2}} \mu_{\tau}^{+}+\varepsilon_{\tau}^{+}, \quad \tau=1,2, \ldots, T \equiv N / \delta, \\
& \mu_{\tau}^{+}=\mu_{\tau-1}^{+}+\eta_{\tau}^{+}, \quad \mu_{0}^{+}=0,
\end{aligned}
$$

where $\left(\varepsilon_{\tau}^{+}, \eta_{\tau}^{+}\right)^{\prime}$ are serially uncorrelated Gaussian disturbances with

$$
\operatorname{Var}\left(\begin{array}{c}
\varepsilon_{\tau}^{+} \\
\eta_{\tau}^{+}
\end{array}\right)=\left(\begin{array}{cc}
\sigma_{\varepsilon}^{2}+\delta^{2} \sigma_{\eta}^{2} / 3 & -\frac{1}{2} \delta \sigma_{\eta}^{2} \\
-\frac{1}{2} \delta \sigma_{\eta}^{2} & \sigma_{\eta}^{2}
\end{array}\right),
$$

and $A_{\tau}=X_{\tau}^{+} \beta^{+}$is a deterministic component. From section 3.3, the LBI statistic is

$$
\bar{L}_{F}(\delta)=\frac{T^{-2} \sum_{\tau=1}^{T}\left(\sum_{j=\tau}^{T} e_{j}^{+}\right)^{2}}{T^{-1} \sum_{\tau=1}^{T} e_{\tau}^{+2}}
$$

where $e_{\tau}^{+}$is the $\tau$-th element of the vector of OLS residuals $\mathbf{e}_{F}$, obtained from regressing the observation vector $\mathbf{y}_{F}=\left(Y_{1}, \ldots, Y_{T}\right)^{\prime}$ on the deterministic variables $\mathbf{X}_{F}=\left(X_{1}^{+\prime}, \ldots, X_{T}^{+\prime}\right)^{\prime}$. Again, (4.7) is the usual form of the LBI test of stationarity for data sampled at constant intervals.

Noticing that the random walk component in the observation equation is scaled by $\delta^{\frac{3}{2}}$, rather than $\delta^{\frac{1}{2}}$ as in the stock variable case, and adapting the previous arguments used for the stock case, it is easily demonstrated that as $\delta \rightarrow 0$ with $N$ fixed, $\bar{L}_{F}(\delta)$ is of $O_{p}(1)$ and consequently the LBI test is not consistent in the case of flow data; cf. Table 1 of section 4.3. 


\subsection{Monte Carlo Simulations}

In this section we use Monte Carlo simulation methods to investigate the finite-sample power properties of the LBI tests of section 3.3. All experiments reported in this paper were programmed using the random number generator of the matrix programming language $\mathrm{Ox}$ 2.20 of Doornik (1998), over 10,000 Monte Carlo replications. All tests were run at the nominal $5 \%$ asymptotic level, although other choices of the nominal level did not alter the results qualitatively.

Specifically, we simulate data according to the discrete-time analogues (2.6)-(2.7) for stock data and (2.9)-(2.10) for flow data over a time span of $N=100$ and setting $\delta_{\tau}=\delta$ constant. Without loss of generality we set $\sigma_{\varepsilon}^{2}=1$ and compute the tests across a range of values of $\sigma_{\eta}^{2}=c^{2} / N^{2}$, for $c=0,2.5,5,10,25$, and of the sampling interval $\delta=1,1 / 2,1 / 4,1 / 6,1 / 12$. In each case the number of observations is therefore $100 / \delta$. Notice, therefore, that the implied signal-to-noise ratios in the discrete-time analogue models for the cases of stock and flow variables will be $\delta c^{2} / 100^{2}$ and $\delta^{2} c^{2} /\left(\frac{1}{3} \delta^{2} c^{2}+100^{2}\right)$, respectively; cf. (4.2)-(4.3).

We may think of this as data from an underlying continuous time process observed over a 100 year span at regular discrete intervals in time corresponding to an annual, biannual, quarterly, bi-monthly and monthly sampling frequency when $\delta=1,1 / 2,1 / 4,1 / 6,1 / 12$, respectively. Without loss of generality, we have set the deterministic kernel $a(t)=0$ in what follows, since our tests are constructed from exact invariant statistics.

Tables $1 \mathrm{a}$ and $1 \mathrm{~b}$ report the empirical rejection frequencies, as functions of $c$ and $\delta$, for the tests which reject for large values of the LBI statistics $\bar{L}_{S}(\delta)$ and $\bar{L}_{F}^{*}(\delta)$, for stock and flow variables respectively. The stock and flow statistics were constructed from the residual vectors $\mathbf{e}_{S}$ and $\mathbf{e}_{F}$ whose elements are the de-meaned $(\xi=1$ in Table 1a) or de-trended ( $\xi=2$ in Table $1 b)$ observations on the observed stock and flow variables respectively. Consequently all of the statistics possess a $V M_{\xi}(1)$ limiting distribution under $H_{0}$ of (2.4); cf. section 3.3. The $5 \%$ asymptotic critical value for the tests is therefore 0.461 for $\xi=1$ and 0.149 for $\xi=2$; see, for example, Harvey (2001).

\section{Tables $1 \mathrm{a}-1 \mathrm{~b}$ about here}

A number of important observations can be drawn from the results presented in Tables 1a and $1 \mathrm{~b}$. Firstly, in the case where $\delta=1$ the power of the tests for stock and flow variables 
are almost identical throughout. This is, of course, to be expected given that the signal-tonoise ratio for stock variables is given by $c^{2} /\left(T^{2} \delta\right)$ while for flow variables it tends to $c^{2} / T^{2}$, as $T$, the number of sample observations, increases; see sections 4.1 and 4.2. Moreover, the observed powers reported in Tables $1 \mathrm{a}$ and $1 \mathrm{~b}$ for $\delta=1$ closely correspond with the limiting power functions of Model A and Model B of Tanaka (1996,p.390), respectively, despite being obtained for the moderately small sample size of $T=100$.

Secondly, for the case of flow variables we see from the results in Tables 1a and 1b that, ceteris paribus, power does not increase as the sampling interval $\delta$ decreases, as predicted by the limiting distribution theory in section 4.2. Indeed, for a given value of $c$, there are virtually no differences in power across the various values of $\delta$ considered.

Thirdly, in the case of stock variables we see that power increases as $\delta$ decreases, other things equal. For example, in Table 1a with $c=5$ the power for $\delta=1$, where we have annual observations on the underlying continuous-time process, is $30.3 \%$ but for data which have been observed monthly throughout the sample the power of the test is dramatically increased to $82.1 \%$. Moreover, as predicted by the limiting distribution theory in section 4.1, the finite sample local power of the test appears closely related to the quantity $c_{S} \equiv c / \sqrt{\delta}$. For example, $c=2.5, \delta=1 / 4$ and $c=5, \delta=1$ both give $c_{S}=5$, while $c=5, \delta=1 / 4$ and $c=10, \delta=1$ both equate to $c_{S}=10$. We see from the results in Tables $1 \mathrm{a}, 1 \mathrm{~b}$ that the power of the stock test for $c=2.5, \delta=1 / 4$ and $c=5, \delta=1$ is very similar, and is also very similar for $c=5, \delta=1 / 4$ and $c=10, \delta=1$.

Finally, notice that, as expected, the simulated powers reported in Table 1a are higher than the corresponding entries in Table 1b. This is due to the usual efficiency loss resulting from the estimation of an extra parameter in the model with a linear trend.

\subsection{Empirical Illustrations}

Figure 1 graphs the quarterly seasonally unadjusted series of the Italian unemployment rate over the period 1980Q1-2003Q4; the data source is Istat, the Italian statistical office. A random walk plus noise model, where a time-varying seasonal component is also included as in Harvey (1989, p. 42), satisfactorily tracks the data, with a standard error of 0.41 and with no evidence of serial correlation in the Kalman filter residuals. The maximum likelihood estimates of $\sigma_{\eta}^{2}$ and $\sigma_{\varepsilon}^{2}$ are 0.1279 and 0.0109 , with signal-to-noise ratio $q=11.73$, 
while the variance of the seasonal component is only 0.0003. The LBI test, applied to the seasonally adjusted data, ${ }^{6}$ provides a sound rejection of the null hypothesis of stationarity: the statistic (4.4) is equal to 1.827 compared with the $10 \%, 5 \%$ and $1 \%$ asymptotic critical values of $0.347,0.461$ and 0.743 , respectively. Even with a (superfluous) non-parametric long-run variance correction of the form considered in KPSS pp.164-65 employed, the null is still rejected at at least the $5 \%$ significance level when a lag truncation of three or less is used in the long-run variance estimator. As the unemployment rate is a stock variable, on the basis of the foregoing theoretical development one would expect, other things equal, less evidence for rejection under the alternative if the stationarity test were to be applied to annual data. Indeed, the LBI statistic applied to the annual data (with no long-run variance correction) takes the greatly reduced value 0.374 , which is now only a borderline rejection at the $10 \%$ significance level. It is also interesting to notice that fitting a random walk plus noise model to the annual data yields $\sigma_{\eta}^{2}=0.6418$ (with $\sigma_{\varepsilon}^{2}$ approximately zero); this is not inconsistent with what one would compute by multiplying by four the variance in the quarterly model; cf. (2.6)-(2.7).

\section{Figures $1-3$ about here}

Consider now Figure 2 which graphs annual and biennial data (the latter divided by two in the graph) on the flow of the Nile over the period 1871-1970. The unit of measurement is cubic metres times $10^{8}$; see Koopman et al. (2000). It is known that the Aswan dam was constructed in 1899 and consequently one has to account for a structural break in the level of the series. The stationarity tests therefore have to be run with a level change in the deterministic component. Critical values for this case are provided in Busetti and Harvey (2001); for a breakpoint fraction equal to 0.3 the $10 \%$ asymptotic critical value is 0.189 . The LBI statistic for the biennial data takes the value 0.086, signalling no evidence that a random walk component is present in the data. Consonant with the large sample theory, doubling the number of observations by running the test on the annual data does not alter our inference; the LBI statistic takes the value 0.089 , which is virtually unchanged from that obtained from the biennial data.

Figure 3 depicts the monthly inflation rate in Japan, computed as first difference of the

\footnotetext{
${ }^{6}$ The data were seasonally adjusted in the context of the Basic Structural Model of Harvey (1989, p. 47), using the inbuilt seasonal adjustment procedure in the STAMP 6.0 package of Koopman et al. (2000).
} 
logarithm of the Consumer's Price Index; the source is the Bank of International Settlements. Inflation can be regarded as a flow variable since it is the first difference of a stock and it is clearly measured with respect to an interval of time. Quarterly inflation can be calculated equivalently as a three-period average of the monthly data or as the three-month differences of the logarithm of the price index. In order to allow for serial dependence in the series, we calculated the statistic of KPSS, Equation (13) p.165 (notice that this is just the LBI statistic of (4.7) with the OLS variance estimator in the denominator replaced by a corresponding long-run variance estimator), on both monthly and quarterly inflation. We computed KPSS statistics over the full sample data (1985-2002, with a time span, $N$, equal to 72 quarters) and over the two sub-samples 1985-1993 and 1994-2002, each of which has $N=36$. The statistics were calculated for two values, $x=4$ and $x=8$ of the lag trucation parameter suggested in KPSS, $\ell=\operatorname{int}\left[x(T / 100)^{\frac{1}{4}}\right], T$ the the number of sample observations. The OLS residuals, $e_{\tau}^{+}$where obtained in each case from regressing the observed data on a set of conventional seasonal dummies, so as to account for the deterministic seasonal fluctuations displayed by the series. Doing so does not affect the limiting null distributions of the resulting statistics vis-à-vis the case where only a constant is included; cf. Phillips and Jin (2002).

For the full sample data there is a rather strong evidence against the null hypothesis of stationarity: for the monthly data with $x=4$ and $x=8$ the KPSS statistic takes the values 0.863 and 0.662 , respectively, which are significant at the $1 \%$ and $5 \%$ significance levels, respectively; for the quarterly data the corresponding outcomes are 0.573 and 0.420 which are significant at the $5 \%$ and $10 \%$ levels, respectively. In contrast, for the first subsample, 1985-1993, the outcomes for the monthly (quarterly) data with $x=4$ and $x=8$ are 0.367 and 0.330 (0.311 and 0.229), while for the second sub-sample, 1994-2002, the corresponding outcomes are 0.282 and 0.283 (0.283 and 0.235$)$, all but the first of which are insignificant at the $10 \%$ level. These outcomes are consistent with the theoretical prediction that the limiting power of the test can only be increased by enlarging the span of the data. Qualitatively similar results were also found for other sub-samples of length $N=36$, so that the observed results are not merely attributable to the particular sample split reported. 


\section{$5 \quad$ Stationarity Tests with Mixed Frequency Data}

As Harvey (1989,p.325) comments, "A series may sometimes consist of observations at two different timing intervals. For example, observations may, at first, only be available on an annual basis, whereas later on they are collected every quarter." In this section we explore the impact of such mixed frequency data on tests of $H_{0}$ of (2.4) against $H_{1}$ of (2.5) for both stock and flow variables. Since both sub-samples include information about the trend, how we handle the mixed frequency data is potentially important for the power properties of the resulting test.

In this section we derive the exact LBI tests for both stock and flow variables in cases where we have a sample of mixed frequency data. We show that the LBI statistics have nonpivotal limiting distributions. We compare the exact LBI tests with simple modifications of these statistics, constructed to have pivotal limiting distributions, and also tests which are constructed from the aggregated data. The former are obtained by applying the statistic $\mathcal{N} \mathcal{M}$ of (1.3) to each of the two sub-samples and adding the resulting statistics together, while the latter are tests which aggregate the more frequently observed data so as to have the same sampling frequency as the data from the first interval; see Harvey (1989,p.310).

In what follows, we suppose that for some $\lambda \in[0,1]$ the first $[\lambda T]$ observations have been made with a sampling frequency of one, possibly annually as in Harvey's example above, while the remaining $T-[\lambda T]$ observations are made with a sampling frequency of $\delta^{-1} \geq 1 ;^{7}$ for example, the case where the first half of the available sample data consists of annual observations and the second half quarterly observations corresponds to $\lambda=0.5$ and $\delta=1 / 4$. The corresponding sequence of intra-observation intervals will therefore be given by

$$
\delta_{\tau}= \begin{cases}1 & 1 \leq \tau \leq[\lambda T] \\ \delta & {[\lambda T]+1 \leq \tau \leq T}\end{cases}
$$

Consequently, the LBI tests for $H_{0}$ of (2.4) against $H_{1}$ of (2.5) for the case of stock and flow variables reject for large values of the statistics given in (3.2) and (3.6) respectively, on

\footnotetext{
${ }^{7}$ One might also consider the case where $\delta^{-1} \leq 1$, that is where observations become available at less frequent intervals after time $[\lambda T]$. The theory which follows is also appropriate to this case but we choose to focus on $\delta^{-1} \geq 1$ because this seems more likely in practice. Moreover, although we have set the sampling frequency in the first sub-sample to be one this also involves no loss of generality.
} 
setting the $\delta_{\tau}$ as above in the formulation thereof. Moreover, notice that

$$
t_{\tau} \equiv \sum_{j=1}^{\tau} \delta_{j}= \begin{cases}\tau & 1 \leq \tau \leq[\lambda T] \\ \delta \tau+(1-\delta)[\lambda T] & {[\lambda T]+1 \leq \tau \leq T}\end{cases}
$$

and thus $N \equiv t_{T}=[\lambda T]+\delta(T-[\lambda T])$.

The limiting distribution of the LBI statistic for the case of a stock variable, $L_{S}$, under $H_{0}$ of (2.4) follows using (3.3), (C.4) and the CMT. Specifically,

$$
L_{S} \Rightarrow \int_{0}^{\lambda} B_{S, \xi}(r)^{2} d r+\delta \int_{\lambda}^{1} B_{S, \xi}(r)^{2} d r .
$$

The form of the limiting process $B_{S, \xi}(r)$ depends on the deterministic component $a(t)$. For $\xi=1$, the constant case, it was demonstrated in section 2.1 that $a_{\tau}=a$ in $(2.6)-(2.7)$, regardless of $\delta_{\tau}$, and hence $\mathbf{e}_{S}$ are simply the de-meaned observations on $y_{\tau}$. Consequently, $B_{S, 1}(r)$ will be a standard Brownian bridge process. For $\xi=2$, the linear trend case, the form of the limiting process $B_{S, 2}(r)$ is more complicated and is given in Appendix $\mathrm{C} 1$.

Turning to the flow variable case, the limiting distribution ${ }^{8}$ of $L_{F}^{*}$ under $H_{0}$ of $(2.4)$ can similarly be established as

$$
L_{F}^{*} \Rightarrow \int_{0}^{\lambda} B_{F, \xi}(r)^{2} d r+\delta \int_{\lambda}^{1} B_{F, \xi}(r)^{2} d r .
$$

Again the form of the limiting process $B_{F, \xi}(r)$ depends on $a(t)$. For $\xi=1$, it follows from the results in section 2.2 that $A_{\tau}=a$ for $1 \leq \tau \leq[\lambda T]$ and $A_{\tau}=a \delta$ for $[\lambda T]+1 \leq \tau \leq T$, in (2.6)-(2.7). It then follows, that the residual vector $\mathbf{e}_{F}$ has $\tau$ th element

$$
e_{\tau, F}=\delta_{\tau}^{-\frac{1}{2}}\left(Y_{\tau}-\delta_{\tau} \sum Y_{\tau} / N\right)= \begin{cases}Y_{\tau}-\sum Y_{\tau} / N & \tau \leq[\lambda T] \\ \delta^{-\frac{1}{2}}\left(Y_{\tau}-\delta \sum Y_{\tau} / N\right) & \tau>[\lambda T]\end{cases}
$$

From which it follows, as demonstrated in Appendix C2, that

$$
B_{F, 1}(r) \equiv \begin{cases}\delta^{\frac{1}{2}}(W(1)-W(\lambda))+(W(\lambda)-W(r))-(\delta(1-\lambda)+\lambda-r) J & r \leq \lambda \\ \delta^{\frac{1}{2}}(W(1)-W(r))-\delta(1-r) J & r>\lambda\end{cases}
$$

where $J \equiv(\lambda+\delta(1-\lambda))^{-1}\left(W(\lambda)+\delta^{1 / 2}(W(1)-W(\lambda))\right)$, and $W(r)$ is a standard Brownian motion. The form of the limiting process $B_{F, 2}(r)$ is more complicated and is given in Appendix C3.

\footnotetext{
${ }^{8}$ Recall from section 3.2 that $L_{F}^{*}$ and $L_{F}$ are asymptotically equivalent.
} 
As is clear from (5.2) and (5.3) the limiting null distributions of the $L_{S}$ and $L_{F}^{*}$ statistics depend in a complicated manner on two nuisance parameters, $\delta$ and $\lambda$. Consequently, asymptotic critical values for the two tests will need to be tabulated across these two parameters. In Tables $2 \mathrm{a}$ and $2 \mathrm{~b}$ we present the upper-tail $1 \%, 5 \%$ and $10 \%$ fractiles from the right members of (5.2) and (5.3), respectively, for $\lambda \in\{0.25,0.50,0.75\}$ and $\delta \in\{1,1 / 2,1 / 4,1 / 6,1 / 12\}$, for both $\xi=1$ (constant) and $\xi=2$ (linear trend). In each case these were calculated via direct simulation (that is, approximating the limit functionals using partial sums of normal random variables) for $T=5000$, using 50000 Monte Carlo replications.

\section{Tables $2 \mathrm{a}-2 \mathrm{~b}$ about here}

There is, however, a simple way of obtaining statistics in both stock and flow cases which have pivotal limiting null distributions in the presence of mixed frequency observations. In what follows the subscript index $i$ is used to denote either the case of a stock variable, $i=S$, or of a flow variable, $i=F$. Define the vectors of OLS residuals $\tilde{\mathbf{e}}_{i}^{*}=\left(\tilde{e}_{1, i}^{*}, \ldots, \tilde{e}_{T, i}^{*}\right)^{\prime}$, $i=S, F$, as follows. For the case of stock variables these are obtained from regressing $y_{\tau}$ on either $\left(1, h_{\tau}(\lambda)\right)^{\prime}$, for the constant case, or, for the linear trend case, $\left(1, \tau, h_{\tau}(\lambda), \tau h_{\tau}(\lambda)\right)^{\prime}$, $\tau=1, \ldots T$, where $h_{\tau}(\lambda) \equiv 1(\tau \leq[\lambda T]), 1(\cdot)$ the usual indicator function. For the case of flow variables the same regressions are computed on replacing $y_{\tau}$ by $Y_{\tau}$.

Using these residuals we then construct the modified statistics

$$
\widehat{L}_{i}^{*}=\frac{\sum_{\tau=1}^{[\lambda T]}\left(\sum_{\kappa=\tau}^{T} \tilde{e}_{\kappa, i}\right)^{2}}{[\lambda T] \sum_{\tau=1}^{[\lambda T]} \tilde{e}_{\tau, i}^{2}}+\frac{\sum_{\tau=[\lambda T]+1}^{T}\left(\sum_{\kappa=\tau}^{T} \tilde{e}_{\kappa, i}\right)^{2}}{(T-[\lambda T]) \sum_{\tau=[\lambda T]+1}^{T} \tilde{e}_{\tau, i}^{2}}, \quad i=S, F .
$$

It is important to notice that for both stock and flow variables, the left member of (5.4) is constructed by applying the statistic $\mathcal{N} \mathcal{M}$ of (1.3) separately to each of the two sub-samples, $\{1, \ldots,[\lambda T]\}$ and $\{[\lambda T]+1, \ldots, T\}$, of stock or flow data and then taking the sum of the two resulting sub-sample statistics. In doing so we are clearly ignoring the information on the sequence of intra-observation intervals $\delta_{\tau}, \tau=1, \ldots, T$.

Since, in all cases considered, $\sum_{\kappa=[\lambda T]+1}^{T} \tilde{e}_{\kappa, i} \equiv 0$, the two terms which appear in the right member of (5.4) are asymptotically independent. Consequently, it trivially follows from the results in section 3 and the CMT that, under $H_{0}$ of $(2.4)$,

$$
\widehat{L}_{i}^{*} \Rightarrow \int_{0}^{1} B_{\xi, 1}(r)^{2} d r+\int_{0}^{1} B_{\xi, 2}(r)^{2} d r, \quad i=F, S
$$


where $B_{\xi, 1}(r)$ and $B_{\xi, 2}(r)$ are independent $V M_{\xi}(1)$ distributions, $\xi=1,2$. By the additive properties of the Cramér-von Mises family of distributions (see, Busetti and Harvey, 2001, p.136), we obtain that the right member of (5.5) is a pivotal $\xi$ th-level Cramér-von Mises distribution with two degrees of freedom; denoted $V M_{\xi}(2), \xi=1,2$. Critical values from the $V M_{1}(2)$ and $V M_{2}(2)$ distributions are tabulated in Nyblom and Harvey (2000) and Harvey (2001).

One might also consider aggregating the second sub-sample of data such that the aggregated observations in the second sub-sample have the same sampling frequency as the data from the first interval. The aggregated stock and flow data are given by $y_{\tau}=y(\tau)$ and $Y_{\tau}=\int_{\tau-1}^{\tau} y(r) d r, \tau=1,2, \ldots, N$, which we collect into the $N$-dimensional vectors $\mathbf{y}_{S}^{*}=\left(y_{1}, y_{2}, \ldots, y_{N}\right)^{\prime}$ and $\mathbf{y}_{F}^{*}=\left(Y_{1}, Y_{2}, \ldots, Y_{N}\right)^{\prime}$, respectively. We then simply apply the standard stationarity test, $\mathcal{N} \mathcal{M}$ of $(1.3)$ to $\mathbf{y}_{S}^{*}$ and $\mathbf{y}_{F}^{*}$, respectively. In what follows we will denote the resulting statistics as $L_{S, 0}$ and $L_{F, 0}^{*}$, respectively. It is trivial to show that both $L_{S, 0}$ and $L_{F, 0}^{*}$ weakly converge under $H_{0}$ of $(2.4)$, as $T \rightarrow \infty$, to $V M_{\xi}(1)$ distributions, $\xi=1,2$. For $\xi=1$ the residuals used in constructing the $L_{S, 0}$ and $L_{F, 0}^{*}$ statistics are the de-meaned $\mathbf{y}_{S}^{*}$ and $\mathbf{y}_{F}^{*}$ respectively, while for $\xi=2$ they are formed as the de-trended $\mathbf{y}_{S}^{*}$ and $\mathbf{y}_{F}^{*}$ respectively.

Both the modified statistics of (5.4) and the statistics based on the aggregated data ignore the infra-period aspect of the data. One might expect that in the case of flow variables these tests will have very similar power properties to the test based on $L_{F}^{*}$ (which is approximately LBI) since, as shown in section 4, power does not increase with the sampling frequency in the case of flow variables. In contrast, the results in section 4.3 would lead us to expect significant power losses from ignoring the infra-period information in the case of stock variables. We now use numerical methods to explore these issues further.

\subsection{Monte Carlo}

In this section we simulate mixed frequency data for $\delta \in\{1,1 / 2,1 / 4,1 / 6,1 / 12\}$, and $\lambda \in\{0.25,0.50,0.75\}$. Using the observed data, $y_{\tau}$ (stock) and $Y_{\tau}$ (flow), we compute the statistics $L_{S}, L_{F}^{*}, \widehat{L}_{S}^{*}$ and $\widehat{L}_{F}^{*}$. The first two statistics are then compared with the asymptotic $5 \%$ critical values from Tables $2 \mathrm{a}$ and $2 \mathrm{~b}$, and the latter two with the upper $5 \%$ point from the $V M_{\xi}(2)$ distribution; that is, 0.748 for a constant level $\xi=1$ and 0.247 for the linear 
trend case $\xi=2$; see, for example, Harvey (2001). In addition we also consider the standard $\mathcal{N} \mathcal{M}$ statistics, denoted $L_{S, 0}$ and $L_{F, 0}^{*}$, applied to the aggregated stock and flow data, $\mathbf{y}_{S}^{*}$ and $\mathbf{y}_{F}^{*}$, as described above.

Specifically, we simulate the local level process (1.1)-(1.2) for $t=1,2, \ldots, 96 / \delta$, across $\delta \in\{1,1 / 2,1 / 4,1 / 6,1 / 12\}$. Taking $\delta=1$ to represent yearly data, we consider the cases of annual observations that become biannual, quarterly, bi-monthly, monthly when $\delta$ assume the values $1 / 2,1 / 4,1 / 6,1 / 12$ respectively. The signal-to-noise ratio is set to $\sigma_{\eta}^{2} / \sigma_{\varepsilon}^{2}=c^{2} /(96 / \delta)^{2}$, for $c=0,2.5,5,10$, in order that we may simulate the power functions of the tests in terms of the magnitude of the local alternative hypothesis in a process generated with constant sampling frequency $\delta^{-1}$.

From this underlying DGP we obtain mixed frequency observations for stock and flow variables, where the first $[\lambda T]$ observations are sampled with unit frequency (annual) and the remaining $T-[\lambda T]$ observations are sampled with frequency $\delta^{-1} \geq 1$. In particular, for each $\delta$, data have been generated with frequency $\delta^{-1}$ and then aggregated only in the first subsample in order to obtain observations with unit frequency. ${ }^{9}$ Notice that $T=96$ in all cases, while $\lambda$ varies among $\{0.25,0.50,0.75\}$.

\section{Tables $3 a-3 c$ about here}

Tables 3a-3c report the empirical rejection frequencies, as functions of $c, \delta$ and $\lambda$, for the tests which reject for large values of the LBI statistics $L_{S}, L_{F}^{*}$, the simplified statistics $\widehat{L}_{S}^{*}, \widehat{L}_{F}^{*}$ and the standard $\mathcal{N} \mathcal{M}$ statistics $L_{S, 0}, L_{F, 0}^{*}$ applied to the aggregated stock and flow data, as explained in the previous section. Notice that the latter two are computed for a number of observations equal to $(\lambda+\delta(1-\lambda)) T \leq T$.

First notice that for $\delta=1$ the results in Tables 3a-3c are much the same, since here the observation frequency is constant throughout the sample. Moreover, here the results for the LBI tests closely resemble the simulated local limiting powers reported in Tables 1a-1b for $\delta=1$. Notice also that in Tables 3a-3c, but not in Tables 1a-1b, the time span $N$ increases as $\delta$ reduces: $N=(\lambda / \delta+1-\lambda) T$. It is therefore no surprise that the simulated power of the tests for both stock and flow turns out to be, in general, an increasing function of the

\footnotetext{
${ }^{9}$ Notice that the Monte Carlo experiments reported in this section involve (partial) temporal aggregation over fixed time intervals and thus are slightly different from those of section 4.3 where data were generated from the exact discrete-time analogues of the underlying continuous-time process.
} 
sample frequency $\delta^{-1}$.

Consider now in particular Table $3 \mathrm{~b}$, where $\lambda=0.50$, that is the case where half of the data are observed on an annual basis and the remaining half at higher frequency. The empirical size, contained in the first 6 rows of the table where $c=0$, is reasonably close to the nominal asymptotic $5 \%$ level for all reported tests. For stock variables the highest power is achieved by using the LBI test $L_{S}$. Here the power loss from ignoring the more finely available observations and running the standard NM test on the aggregated data, namely using the statistic $L_{S, 0}$, is not negligible; for example, in the case of constant level with $c=2.5$ and $\delta=1 / 12$ the use of the LBI test yields power of $42.1 \%$, as against $26.8 \%$ when using the corresponding aggregated data. Despite using all available data, the modified test $\widehat{L}_{S}$ which combines the evidence in the two subsamples is less powerful than $\widehat{L}_{S, 0}$, with power of $21.3 \%$ in the foregoing example. This is attributable to the efficiency loss incurred by having to estimate additional parameters. For flow variables, on the other hand, the power losses from using aggregate data appear negligible. In the example used above, where $c=2.5$ and $\delta=1 / 12$, the rejection frequencies are $77.8 \%$ for the LBI test $L_{F}^{*}$ and $74.5 \%$ for the NM test $L_{F, 0}^{*}$ for the case of constant level. Again, and for the same reason, lower power, $65.9 \%$, is obtained by running the modified test $\widehat{L}_{F}$.

The results for the linear trend case are qualitatively similar to those reported for a constant level, although for each configuration of $\delta, \lambda$ and $c$ power is smaller. This is analogous to what happens in the standard NM tests; cf. KPSS.

The results reported in Tables $3 \mathrm{a}$ and $3 \mathrm{c}$, where $\lambda$ is 0.25 and 0.75 respectively, are also broadly similar to the corresponding results for $\lambda=0.50$. The main difference is the lower (respectively higher) power than in Table 3b. This can be easily explained by looking at the span of the data $N=(\lambda / \delta+1-\lambda) T$, which, for $\delta<1$, is increasing in $\lambda$.

In summary, the Monte Carlo results strongly suggest using the LBI test if the variables are stock; the critical values for $\lambda \in\{0.25,0.50,0.75\}$ are provided in Tables $2 \mathrm{a}$ and $2 \mathrm{~b}$, for other values of $\lambda$ they can be obtained from the authors on request. For flow variables, on the other hand, it appears that near-efficient tests can be obtained even when one ignores the more finely available observations, since, as we have already seen, the limiting power properties of the tests for flow variables depend on the span of the data and not the number of sample observations. 


\section{Conclusions}

Using a continuous-time framework in this paper we have derived locally best invariant (LBI) stationarity tests for both stock and flow data available at potentially irregularly spaced points in time. The resulting tests were shown to differ between stock and flow variables. The special case of mixed frequency data was analysed in detail with asymptotic critical values and a power study provided. Our results suggested that tests based on statistics which ignore the infra-period aspect of the data, such as those constructed from aggregated data, involve rather small losses in efficiency, relative to the LBI test, for the case of flow variables, but can incur significant efficiency losses when dealing with stock variables. We have also demonstrated that where the data are observed at regular intervals throughout the sample the LBI tests for stock and flow data reduce to the form of the standard stationarity test of, inter alia, NM applied to the observed stock and flow data, respectively. These statistics were shown to have Cramér-von Mises limiting null distributions. For regularly sampled data we also demonstrated that the asymptotic local power of the LBI test increases with the sampling frequency in the case of stock variables but not for flow variables. Moreover, for a fixed time span the LBI test for stock variables was shown to be consistent against a fixed alternative as the sampling frequency increased to infinity. This was shown not to be true in the case of flow variables.

Although we have focused on issues concerned with testing against a unit root at frequency zero, the analysis of this paper can be extended to the seasonal stationarity tests developed in, inter alia, Canova and Hansen (1995). As an example, if one had mixed frequency data observed first annually then subsequently quarterly it is clear that only the second sub-sample contains information useful in constructing tests against seasonal frequency unit roots. However, if our data were available first quarterly and then monthly, information on the seasonal spectral frequencies $\pi$ and $\pi / 2(3 \pi / 2)$ would be contained in both sub-samples. Consequently, seasonal unit root tests for mixed frequency data, akin to those developed in section 5 , can be constructed to be more powerful (in the case of stock variables) than those based on data where the monthly data is aggregated to quarterly data. Moreover, by adopting a continuous-time framework one can also show that, in the case of stock variables, tests against, for example, a pair of complex conjugate unit roots at frequency $\pi / 2(3 \pi / 2)$ will be consistent against fixed alternatives for the case where a fixed 
data span is available but where the sampling frequency tends to infinity as a multiple of four.

\section{Appendix A}

From (2.2)-(2.1) we have that

$$
\begin{aligned}
Y_{\tau} & =\int_{t_{\tau-1}}^{t_{\tau}} y(t) d t \\
& =\int_{t_{\tau-1}}^{t_{\tau}} a(t) d t+\int_{t_{\tau-1}}^{t_{\tau}}\left(\int_{0}^{t} \xi_{2}(d s)\right) d t+\int_{t_{\tau-1}}^{t_{\tau}} \xi_{1}(d t) \\
& =A_{\tau}+\int_{t_{\tau-1}}^{t_{\tau}}\left(\mu\left(t_{\tau}\right)-\int_{t}^{t_{\tau}} \xi_{2}(d s)\right) d t+\int_{t_{\tau-1}}^{t_{\tau}} \xi_{1}(d t) \\
& =A_{\tau}+\delta_{\tau} \mu\left(t_{\tau}\right)-\int_{t_{\tau-1}}^{t_{\tau}} \int_{t}^{t_{\tau}} \xi_{2}(d s) d t+\int_{t_{\tau-1}}^{t_{\tau}} \xi_{1}(d t),
\end{aligned}
$$

where integrals are defined in the wide sense, with the exception of those taken with respect to the random measure; see Bergstrom (1984). Defining $\mu_{\tau}=\mu\left(t_{\tau}\right)$, and $\varepsilon_{\tau}^{*}=\int_{t_{\tau-1}}^{t_{\tau}} \xi_{1}(d t)-$ $\int_{t_{\tau-1}}^{t_{\tau}} \int_{t}^{t_{\tau}} \xi_{2}(d s) d t$, write $(\mathrm{A} .1)$ as

$$
Y_{\tau}=A_{\tau}+\delta_{\tau} \mu_{\tau}+\varepsilon_{\tau}^{*}
$$

where the transition equation is given by

$$
\mu_{\tau}=\mu_{\tau-1}+\eta_{\tau}
$$

with $\eta_{\tau} \equiv \int_{t_{\tau-1}}^{t_{\tau}} \xi_{2}(d t)$.

Using, for example, Bergstrom $(1984,1986)$, we first have that $E\left[\int_{t_{\tau-1}}^{t_{\tau}} \xi_{i}(d t)\right]=0, i=1,2$. Moreover,

$$
\begin{aligned}
E\left[\int_{t_{\tau-1}}^{t_{\tau}} \xi_{1}(d t)\right]^{2} & =\left(t_{\tau}-t_{\tau-1}\right) \sigma_{\varepsilon}^{2} \\
& =\delta_{\tau} \sigma_{\varepsilon}^{2}
\end{aligned}
$$

and, similarly, $E\left[\eta_{\tau}\right]^{2}=\delta_{\tau} \sigma_{\eta}^{2}$, which establishes the result for $\operatorname{Var}\left(\eta_{\tau}\right)$. Notice also that $\left\{\eta_{\tau}\right\}$ is serially independent.

The result for $\operatorname{Var}\left(\varepsilon_{\tau}^{*}\right)$ follows from (A.2) and noting that

$$
E\left[\int_{t_{\tau-1}}^{t_{\tau}} \int_{t}^{t_{\tau}} \xi_{2}(d s) d t\right]^{2}=E\left[\delta_{\tau} \eta_{\tau}-\int_{t_{\tau-1}}^{t_{\tau}}\left(\int_{t_{\tau-1}}^{t} \xi_{2}(d s)\right) d t\right]^{2}
$$




$$
\begin{aligned}
= & \delta_{\tau}^{3} \sigma_{\eta}^{2}+\int_{t_{\tau-1}}^{t_{\tau}} \int_{t_{\tau-1}}^{t_{\tau}} E\left[\int_{t_{\tau-1}}^{t} \xi_{2}(d s) \int_{t_{\tau-1}}^{v} \xi_{2}(d s)\right] d t d v \\
& -2 \delta_{\tau} E\left[\left(\int_{t_{\tau-1}}^{t_{\tau}} \xi_{2}(d s)\right) \int_{t_{\tau-1}}^{t_{\tau}}\left(\int_{t_{\tau-1}}^{t} \xi_{2}(d s)\right) d t\right] \\
= & \delta_{\tau}^{3} \sigma_{\eta}^{2}+\sigma_{\eta}^{2} \int_{0}^{\delta_{\tau}} \int_{0}^{\delta_{\tau}} \min (t, v) d t d v \\
& -2 \delta_{\tau} \int_{t_{\tau-1}}^{t_{\tau}}\left(\int_{t_{\tau-1}}^{t} E\left[\xi_{2}(d s)\right]^{2}\right) d t \\
= & \delta_{\tau}^{3} \sigma_{\eta}^{2}+\frac{1}{3} \delta_{\tau}^{3} \sigma_{\eta}^{2}-2 \delta_{\tau} \sigma_{\eta}^{2} \int_{0}^{\delta_{\tau}} t d t \\
= & \frac{1}{3} \delta_{\tau}^{3} \sigma_{\eta}^{2} .
\end{aligned}
$$

Again notice that $\left\{\varepsilon_{\tau}^{*}\right\}$ is serially independent. Finally,

$$
\begin{aligned}
\operatorname{Cov}\left(\eta_{\tau}, \varepsilon_{\tau}^{*}\right)= & -E\left[\eta_{\tau}\left(\int_{t_{\tau-1}}^{t_{\tau}} \int_{t}^{t_{\tau}} \xi_{2}(d s) d t\right)\right] \\
& -E\left[\eta_{\tau} \int_{t_{\tau-1}}^{t_{\tau}}\left(\int_{t_{\tau-1}}^{t_{\tau}} \xi_{2}(d t)-\int_{t_{\tau-1}}^{t} \xi_{2}(d s)\right) d t\right] \\
= & -E\left[\eta_{\tau}^{2} \int_{t_{\tau-1}}^{t_{\tau}} d t-\eta_{\tau} \int_{t_{\tau-1}}^{t_{\tau}}\left(\int_{t_{\tau-1}}^{t} \xi_{2}(d s)\right) d t\right] \\
= & -\left[\delta_{\tau}^{2}-\int_{t_{\tau-1}}^{t_{\tau}}\left(t-t_{\tau-1}\right) d t\right] \sigma_{\eta}^{2} \\
= & -\left[\delta_{\tau}^{2}-\frac{1}{2}\left(t_{\tau}^{2}-t_{\tau-1}^{2}\right)+\delta_{\tau} t_{\tau-1}\right] \sigma_{\eta}^{2} \\
= & -\frac{1}{2} \delta_{\tau}^{2} \sigma_{\eta}^{2},
\end{aligned}
$$

while all non-contemporaneous covariances are zero.

\section{Appendix B}

From (2.9)-(2.10) the data generating process for $Y_{\tau}^{*} \equiv Y_{\tau}-A_{\tau}$ is given by $Y_{\tau}^{*}=\delta_{\tau} \mu_{\tau}+\varepsilon_{\tau}^{*}=$ $\delta_{\tau}\left(\sum_{j=1}^{\tau} \eta_{j}\right)+\varepsilon_{\tau}^{*}$. Consequently,

$$
\begin{aligned}
E\left(Y_{\tau}^{* 2}\right) & =\delta_{\tau}^{2} E\left(\sum_{j=1}^{\tau} \eta_{j}\right)^{2}+E\left(\varepsilon_{\tau}^{*}\right)^{2}+2 \delta_{\tau} E\left(\varepsilon_{\tau}^{*} \eta_{\tau}\right) \\
& =\delta_{\tau}^{2} \sigma_{\eta}^{2} t_{\tau}+\left(\delta_{\tau}^{3} \sigma_{\eta}^{2} / 3+\delta_{\tau} \sigma_{\varepsilon}^{2}\right)-\delta_{\tau}^{3} \sigma_{\eta}^{2}
\end{aligned}
$$


and, hence,

$$
E\left(\delta_{\tau}^{-1} Y_{\tau}^{* 2}\right)=\sigma_{\varepsilon}^{2}\left(1+q \delta_{\tau} t_{\tau}-2 / 3 q \delta_{\tau}^{2}\right)
$$

Similarly, for $\tau<s$,

$$
\begin{aligned}
E\left(Y_{\tau}^{*} Y_{s}^{*}\right) & =\delta_{\tau} \delta_{s} E\left(\sum_{j=1}^{\tau} \eta_{j} \sum_{i=1}^{s} \eta_{i}\right)+E\left(\varepsilon_{\tau}^{*} \delta_{s} \sum_{i=1}^{s} \eta_{i}\right) \\
& =\delta_{\tau} \delta_{s} \sigma_{\eta}^{2} t_{\tau}-1 / 2 \delta_{s} \delta_{\tau}^{2} \sigma_{\eta}^{2}
\end{aligned}
$$

and, consequently,

$$
E\left(\delta_{\tau}^{-1 / 2} Y_{\tau}^{*} Y_{s}^{*} \delta_{s}^{-1 / 2}\right)=\sigma_{\varepsilon}^{2}\left(q \delta_{\tau}^{1 / 2} \delta_{s}^{1 / 2} t_{\tau}-1 / 2 q \delta_{s}^{1 / 2} \delta_{\tau}^{3 / 2}\right)
$$

\section{Appendix C: Limiting distributions of LBI statistics for mixed fre- quency data}

\section{C1: Stock variables with linear trend.}

Under $H_{0}$ of $(2.4)$ the discrete-time model may be written as

$$
y_{\tau}=a+b z_{\tau}+\varepsilon_{\tau}, \quad \varepsilon_{\tau} \sim N I I D\left(0, \sigma_{\varepsilon}^{2}\right),
$$

where

$$
z_{\tau}= \begin{cases}\tau & \tau \leq[\lambda T], \\ \delta \tau+(1-\delta)[\lambda T] & \tau>[\lambda T] .\end{cases}
$$

Let $\widehat{a}, \widehat{b}$ denote the OLS estimators from (C.1). Using the following limiting results (see also Busetti and Harvey, 2001, p.146)

$$
\begin{aligned}
T^{-2} \sum_{\tau=1}^{T} z_{\tau} & \stackrel{p}{\rightarrow} \frac{1}{2} \delta(1-\lambda)^{2}+\frac{1}{2} \lambda(2-\lambda) \equiv k_{1} \\
T^{-3} \sum_{\tau=1}^{T} z_{\tau}^{2} & \stackrel{p}{\rightarrow} \frac{1}{3}(1-\lambda)^{3} \delta^{2}+\lambda(1-\lambda)^{2} \delta+\frac{1}{3} \lambda^{2}(3-2 \lambda) \equiv k_{2} \\
T^{-\frac{1}{2}} \sum_{\tau=1}^{[T r]} \varepsilon_{\tau} / \sigma_{\varepsilon} & \Rightarrow W(r), \quad r \in[0,1] \\
T^{-\frac{3}{2}} \sum_{\tau=1}^{T} z_{\tau} \varepsilon_{\tau} / \sigma_{\varepsilon} & \Rightarrow \int_{0}^{\lambda} r d W(r)+\delta \int_{\lambda}^{1} r d W(r)+\lambda(1-\delta)(W(1)-W(\lambda)) \equiv J^{*}(
\end{aligned}
$$

where $W(r), r \in[0,1]$, is a standard Brownian motion process, it is straightforward to establish that

$$
\left(\begin{array}{c}
T^{\frac{1}{2}}(\widehat{a}-a) \\
T^{\frac{3}{2}}(\widehat{b}-b)
\end{array}\right) \Rightarrow \frac{\sigma_{\varepsilon}}{k_{3}}\left(\begin{array}{c}
k_{2} W(1)-k_{1} J^{*} \\
-k_{1} W(1)+J^{*}
\end{array}\right)
$$


where $k_{3} \equiv k_{2}-k_{1}^{2}$.

Now, let $e_{\tau}=\varepsilon_{\tau}-(\widehat{a}-a)-z_{\tau}(\widehat{b}-b), \tau=1, \ldots, T$, denote the corresponding OLS residuals from (C.1). Using the foregoing limiting results and applications of the CMT we obtain that

$$
\begin{aligned}
T^{-\frac{1}{2}} \sum_{\tau=1}^{[T r]} e_{\tau} / \sigma_{\varepsilon}= & T^{-\frac{1}{2}} \sum_{\tau=1}^{[T r]} \varepsilon_{\tau} / \sigma_{\varepsilon}-T^{\frac{1}{2}}(\widehat{a}-a)[T r] /\left(T \sigma_{\varepsilon}\right) \\
& \quad-T^{-2} \sum_{\tau=1}^{[T r]} z_{\tau} T^{\frac{3}{2}}(\widehat{b}-b) / \sigma_{\varepsilon} \\
\Rightarrow & W(r)-r\left(k_{2} W(1)-k_{1} J^{*}\right) / k_{3}-\frac{1}{2} m(r)\left(J^{*}-k_{1} W(1)\right) / k_{3} \equiv B_{S, 2}(r)
\end{aligned}
$$

where

$$
m(r)= \begin{cases}r^{2} & r \leq \lambda, \\ \lambda^{2}+\delta\left(r^{2}-\lambda^{2}\right)+2 \lambda(1-\delta)(r-\lambda) & r>\lambda .\end{cases}
$$

Notice that, since there is a constant term in the regression, $\sum_{j=1}^{T} e_{j}=0$ and thus $\sum_{\tau=1}^{T}\left(\sum_{j=\tau}^{T} e_{j}\right)^{2}$ $=\sum_{\tau=1}^{T}\left(\sum_{j=1}^{\tau} e_{j}\right)^{2}$. Since $T^{-1} \sum_{\tau=1}^{T} e_{\tau}^{2} \stackrel{p}{\rightarrow} \sigma_{\varepsilon}^{2}$, the stated limiting distribution, (5.2), then follows immediately using applications of the CMT. Notice that for $\lambda=0,1$ and/or $\delta=1$, $B_{S, 2}(r)$ reduces to a standard second level Brownian bridge process.

\section{C2: Flow variables with constant level.}

Under $H_{0}$ of $(2.4)$ the discrete-time model may be written as

$$
\delta_{\tau}^{-\frac{1}{2}} Y_{\tau}=a z_{\tau}+\varepsilon_{\tau}, \quad \varepsilon_{\tau} \sim N I I D\left(0, \sigma_{\varepsilon}^{2}\right),
$$

where

$$
z_{\tau}= \begin{cases}1 & \tau \leq[\lambda T] \\ \delta^{\frac{1}{2}} & \tau>[\lambda T]\end{cases}
$$

The OLS estimator $\widehat{a}$ from (C.6) therefore satisfies

$$
\widehat{a}-a=\frac{\sum_{\tau=1}^{T} z_{\tau} \varepsilon_{\tau}}{\sum_{\tau=1}^{T} z_{\tau}^{2}}=\frac{\sum_{\tau=1}^{[\lambda T]} \varepsilon_{\tau}+\delta^{1 / 2} \sum_{\tau=[\lambda T]+1}^{T} \varepsilon_{\tau}}{[\lambda T]+\delta(T-[\lambda T])} .
$$

Proceeding as in Appendix C1, it is straightforward to show that

$$
T^{\frac{1}{2}}(\widehat{a}-a) \Rightarrow \sigma_{\varepsilon} J
$$


where $J=(\lambda+\delta(1-\lambda))^{-1}\left(W(\lambda)+\delta^{1 / 2}(W(1)-W(\lambda))\right)$, and $W(r), r \in[0,1]$ is a standard Brownian motion process. Routine algebra establishes that

$$
\begin{aligned}
L_{F}^{*} & =\frac{\mathbf{e}_{F}^{\prime} \mathbf{D}^{\frac{1}{2}} \mathbf{V}_{\delta} \mathbf{D}^{\frac{1}{2}} \mathbf{e}_{F}}{T \mathbf{e}_{F}^{\prime} \mathbf{e}_{F}}=\frac{T^{-2} \sum_{\tau=1}^{T} \delta_{\tau}\left(\sum_{j=\tau}^{T} e_{j} \delta_{j}^{\frac{1}{2}}\right)^{2}}{T^{-1} \sum_{\tau=1}^{T} e_{\tau}^{2}} \\
& =\frac{T^{-2}\left(\sum_{\tau=1}^{[\lambda T]}\left(\sum_{j=\tau}^{T} e_{j} \delta_{j}^{\frac{1}{2}}\right)^{2}+\delta \sum_{\tau=[\lambda T]+1}^{T}\left(\sum_{j=\tau}^{T} e_{j} \delta_{j}^{\frac{1}{2}}\right)^{2}\right)}{T^{-1} \sum_{\tau=1}^{T} e_{\tau}^{2}}
\end{aligned}
$$

where $e_{\tau}=\varepsilon_{\tau}-z_{\tau}(\widehat{a}-a), \tau=1, \ldots, T$, are the OLS residuals from (C.6). Using (C.8) and applications of the CMT it is straightforward but tedious to demonstrate that

$$
T^{-\frac{1}{2}} \sum_{\tau=1}^{[T r]} e_{\tau} \delta_{\tau}^{\frac{1}{2}} \Rightarrow \sigma_{\varepsilon} B_{F, 1}(r),
$$

where $B_{F, 1}(r)$ is as defined in the main text. Notice that for $\lambda=0,1$ and/or $\delta=1, B_{F, 1}(r)$ reduces to a standard Brownian bridge process. The stated result then follows directly from (C.9), (C.10), applications of the CMT, and the fact that $T^{-1} \sum_{\tau=1}^{T} e_{\tau}^{2} \stackrel{p}{\rightarrow} \sigma_{\varepsilon}^{2}$.

\section{C3: Flow variables with linear trend.}

Under $H_{0}$ of (2.4) the discrete-time model may be written as

$$
\delta_{\tau}^{-\frac{1}{2}} Y_{\tau}=a z_{1, \tau}+b z_{2, \tau}+\varepsilon_{\tau}, \quad \varepsilon_{\tau} \sim \operatorname{NID}\left(0, \sigma_{\varepsilon}^{2}\right),
$$

where

$$
\begin{gathered}
z_{1, \tau}= \begin{cases}1 & \tau \leq[\lambda T], \\
\delta^{\frac{1}{2}} & \tau>[\lambda T],\end{cases} \\
z_{2, \tau}= \begin{cases}\tau-\frac{1}{2} & \tau \leq[\lambda T], \\
\delta^{\frac{3}{2}}\left(\tau-\frac{1}{2}\right)+\delta^{\frac{1}{2}}(1-\delta)[\lambda T] & \tau>[\lambda T] .\end{cases}
\end{gathered}
$$

Using the following limiting results (see also Busetti and Harvey, 2001, p.146)

$$
\begin{aligned}
T^{-1} \sum z_{1, \tau}^{2} & \stackrel{p}{\rightarrow} \lambda+\delta(1-\lambda) \equiv k_{1} \\
T^{-2} \sum z_{1, \tau} z_{2, \tau} & \stackrel{p}{\rightarrow} \frac{1}{2}\left(\lambda^{2}+\delta^{2}\left(1-\lambda^{2}\right)+2 \delta \lambda(1-\delta)(1-\lambda)\right) \equiv k_{2} \\
T^{-3} \sum z_{2, \tau}^{2} & \stackrel{p}{\rightarrow} \frac{1}{3}\left(\lambda^{3}+\delta^{3}\left(1-\lambda^{3}\right)+3 \delta \lambda(1-\delta)(1-\lambda)(\lambda+\delta)\right) \equiv k_{3} \\
T^{-\frac{1}{2}} \sum z_{1, \tau} \varepsilon_{\tau} / \sigma_{\varepsilon} & \Rightarrow W(\lambda)+\delta^{\frac{1}{2}}(W(1)-W(\lambda)) \equiv J_{1} \\
T^{-\frac{3}{2}} \sum z_{2, \tau} \varepsilon_{\tau} / \sigma_{\varepsilon} & \Rightarrow \int_{0}^{\lambda} r d W(r)+\delta^{\frac{3}{2}} \int_{\lambda}^{1} r d W(r)+\delta^{\frac{1}{2}}(1-\delta) \lambda(W(1)-W(\lambda)) \equiv J_{2}
\end{aligned}
$$


where $W(r), r \in[0,1]$, is a standard Brownian motion process, it is straightforward to demonstrate that

$$
\left(\begin{array}{c}
T^{\frac{1}{2}}(\widehat{a}-a) \\
T^{\frac{3}{2}}(\widehat{b}-b)
\end{array}\right) \Rightarrow \frac{\sigma_{\varepsilon}}{k_{4}}\left(\begin{array}{c}
k_{3} J_{1}-k_{2} J_{2} \\
-k_{2} J_{1}+k_{1} J_{2}
\end{array}\right)
$$

where $k_{4}=k_{1} k_{3}-k_{2}^{2}$.

Now let $e_{\tau}=\varepsilon_{\tau}-z_{1, \tau}(\widehat{a}-a)-z_{2, \tau}(\widehat{b}-b), \tau=1, \ldots, T$, denote the OLS residuals from (C.11). Consequently, from the foregoing limiting results and applications of the CMT, we obtain that

$$
\begin{aligned}
T^{-\frac{1}{2}} \sum_{\tau=[T r]}^{T} e_{\tau} \delta_{\tau}^{\frac{1}{2}} / \sigma_{\varepsilon}= & T^{-\frac{1}{2}} \sum_{\tau=[T r]}^{T} \varepsilon_{\tau} \delta_{\tau}^{\frac{1}{2}} / \sigma_{\varepsilon}-T^{-1} \sum_{\tau=[T r]}^{T} z_{1, \tau} \delta_{\tau}^{\frac{1}{2}} T^{\frac{1}{2}}(\widehat{a}-a) / \sigma_{\varepsilon} \\
& -T^{-2} \sum_{\tau=[T r]}^{T} z_{2, \tau} \delta_{\tau}^{\frac{1}{2}} T^{\frac{3}{2}}(\widehat{b}-b) / \sigma_{\varepsilon} \\
\Rightarrow & B_{F, 2}(r),
\end{aligned}
$$

where

$$
B_{F, 2}(r) \equiv\left\{\begin{array}{l}
\delta^{\frac{1}{2}}(W(1)-W(\lambda))+(W(\lambda)-W(r)) \\
-\left(f_{a}(\lambda)+\lambda-r\right) J_{a}-\left(f_{b}(\lambda)+\frac{1}{2}\left(\lambda^{2}-r^{2}\right)\right) J_{b}, \quad r \leq \lambda \\
\delta^{\frac{1}{2}}(W(1)-W(r))-f_{a}(r) J_{a}-f_{b}(r) J_{b}, \quad r>\lambda
\end{array}\right.
$$

and where $f_{a}(r)=\delta(1-r), f_{b}(r)=\frac{1}{2} \delta^{2}\left(1-r^{2}\right)+\lambda \delta(1-\delta)(1-r), J_{a}=k_{4}^{-1}\left(k_{3} J_{1}-k_{2} J_{2}\right)$, $J_{b}=-k_{4}^{-1}\left(k_{2} J_{1}-k_{1} J_{2}\right)$. The stated result then follows directly from (C.9), where $e_{\tau}, \tau=$ $1, \ldots, T$, in (C.9) are now the OLS residuals from (C.11), applications of the CMT, and the fact that $T^{-1} \sum_{\tau=1}^{T} e_{\tau}^{2} \stackrel{p}{\rightarrow} \sigma_{\varepsilon}^{2}$. Again, notice that for $\lambda=0,1$ and/or $\delta=1, B_{F, 2}(r)$ reduces to a standard second level Brownian bridge process.

\section{References}

[1] Bailey, R.W. and A.M.R. Taylor, 2002, An optimal test against a random walk component in a non-orthogonal unobserved components model, The Econometrics Journal 5, 520-532.

[2] Bergstrom, A.R., 1983, Gaussian estimation of structural parameters in higher order continuous time dynamic models, Econometrica 51, 117-152. 
[3] Bergstrom, A.R., 1984, Continuous time stochastic models and issues of aggregation over time, in Z. Griliches and M. Intriligator (eds.), Handbook of Econometrics, Amsterdam, North Holland, Vol. 2, pp. 1145-1212.

[4] Bergstrom, A.R., 1985, The estimation of parameters in nonstationary higher-order continuous-time dynamic models, Econometric Theory 1, 369-385.

[5] Bergstrom, A.R., 1986, The estimation of open higher-order continuous-time dynamic models with mixed stock and flow data, Econometric Theory 2, 350-373.

[6] Busetti, F. and A.C. Harvey, 2001, Testing for the presence of a random walk in series with structural breaks, Journal of Time Series Analysis 22, 127-50.

[7] Canova, F. and B.E. Hansen, 1995, Are seasonal patterns constant over time? A test for seasonal stability, Journal of Business and Economic Statistics 2, 292-349.

[8] Chambers, M.J., 2004, Testing for unit roots with flow data and varying sampling frequency, Journal of Econometrics, forthcoming.

[9] Chambers, M.J. and J. McGarry, 2002, Modelling cyclical behavior with differentialdifference equations in an unobserved components framework, Econometric Theory 18, $387-419$.

[10] Comte, F., 1999, Discrete and continuous time cointegration, Journal of Econometrics $88,207-226$.

[11] Doornik J.A., 1998, Object-oriented matrix programming using Ox 2.0, London, Timberlake Consultants Press.

[12] Harvey, A.C., 1989, Forecasting, structural time series models and the Kalman filter, Cambridge University Press: Cambridge.

[13] Harvey, A.C., 2001, Testing in unobserved components models, Journal of Forecasting $20,1-19$.

[14] Harvey, A.C. and J.H. Stock, 1988, Continuous time autoregressive models with common stochastic trends, Journal of Economic Dynamics and Control 12, 365-384. 
[15] Harvey, A.C. and J.H. Stock, 1989, Estimating integrated higher-order continuous time autoregressions with an application to money-income causality, Journal of Econometrics $42,319-336$.

[16] Harvey, A.C. and J.H. Stock, 1993, Estimation, smoothing, interpolation, and distribution for structural time-series models in continuous time, in P.C.B. Phillips (ed.), Models, Methods, and Applications of Econometrics: Essays in Honour of A.R. Bergstrom, Oxford: Blackwell.

[17] Imhof, J.P., 1961, Computing the distribution of quadratic forms in normal variables, Biometrika 48, 419-426.

[18] King, M.L. and G.H. Hillier, 1985, Locally best invariant tests of the error covariance matrix of the linear regression model, Journal of the Royal Statistical Society (Series B) $47,98-102$.

[19] Koopman, S.J., A.C. Harvey, J. A. Doornik and N. Shephard, 2000, STAMP 6.0, Structural Time Series Analyser, Modeller and Predictor, London: Chapman and Hall.

[20] Kwiatkowski, D., P.C.B. Phillips, P. Schmidt and Y. Shin, 1992, Testing the null hypothesis of stationarity against the alternative of a unit root: how sure are we that economic time series have a unit root?, Journal of Econometrics 54, 159-178.

[21] Nabeya, S. and K. Tanaka, 1988, Asymptotic theory of a test for the constancy of regression coefficients against the random walk alternative, Annals of Statistics 16, 218235.

[22] Ng, S., 1995, Testing for unit roots in flow data sampled at different frequencies, Economics Letters 47, 237-242.

[23] Nishino, H., 2002, Stationarity test for data with missing observations, unpublished manuscript, Faculty of Law and Economics, Chiba University, Japan.

[24] Nyblom, J., 1986, Testing for deterministic linear trend in time series, Journal of the American Statistical Association 81, 545-549. 
[25] Nyblom, J. and A.C. Harvey, 2000, Tests of common stochastic trends, Econometric Theory 16, 176-199.

[26] Nyblom, J. and T. Mäkeläinen, 1983, Comparisons of tests for the presence of random walk coefficients in a simple linear model, Journal of the American Statistical Association $78,856-864$.

[27] Perron, P., 1991, Test consistency with varying sampling frequency, Econometric Theory 7, 341-368.

[28] Phillips, P.C.B., 1987, Time series regression with a unit root, Econometrica 55, 277-301.

[29] Phillips, P.C.B., 1991, Error correction and long-run equilibrium in continuous time, Econometrica 59, 967-980.

[30] Phillips, P.C.B. and Z. Xiao, 1998, A primer on unit root testing, Journal of Economic Surveys $12,423-470$.

[31] Stock, J.H., 1994, Unit roots, structural breaks and trends, in R.F. Engle and D.L. McFadden (eds.), Handbook of Econometrics 4, Elsevier Science, 2739-2840.

[32] Tanaka, K., 1996, Time series analysis: nonstationary and noninvertible distribution theory, Wiley: New York.

[33] Wymer, C., 1993, Estimation of nonlinear continuous-time models from discrete data, in P.C.B. Phillips (ed.), Models, Methods and Applications of Econometrics: Essays in Honour of A.R. Bergstrom, Oxford: Blackwell. 
Table 1a: Simulated rejection probabilities $(\times 100)$ : constant level case.

\begin{tabular}{llccccc}
\hline \hline & & \multicolumn{5}{c}{$\delta$} \\
& & 1 & $1 / 2$ & $1 / 4$ & $1 / 6$ & $1 / 12$ \\
\hline$c=0$ & $\bar{L}_{S}(\delta)$ & 5.3 & 5.3 & 5.1 & 4.7 & 4.7 \\
& $\bar{L}_{F}^{*}(\delta)$ & 5.3 & 5.3 & 5.1 & 4.7 & 4.7 \\
\hline \multirow{2}{*}{$c=2.5$} & $\bar{L}_{S}(\delta)$ & 12.9 & 20.3 & 31.7 & 39.4 & 54.8 \\
& $\bar{L}_{F}^{*}(\delta)$ & 12.8 & 13.1 & 13.0 & 12.8 & 12.7 \\
\hline \multirow{2}{*}{$c=5$} & $\bar{L}_{S}(\delta)$ & 30.3 & 45.5 & 60.5 & 69.5 & 82.1 \\
& $\bar{L}_{F}^{*}(\delta)$ & 30.2 & 31.7 & 31.7 & 31.0 & 31.3 \\
\hline \multirow{2}{*}{$c=10$} & $\bar{L}_{S}(\delta)$ & 58.1 & 74.4 & 85.8 & 91.0 & 96.5 \\
& $\bar{L}_{F}^{*}(\delta)$ & 58.1 & 60.8 & 60.6 & 60.4 & 60.8 \\
\hline \multirow{2}{*}{$c=25$} & $\bar{L}_{S}(\delta)$ & 88.3 & 95.5 & 98.8 & 99.6 & 99.9 \\
& $\bar{L}_{F}^{*}(\delta)$ & 88.1 & 90.4 & 90.9 & 91.4 & 91.6 \\
\hline \hline
\end{tabular}

Table 1b: Simulated rejection probabilities $(\times 100)$ : linear trend case.

\begin{tabular}{|c|c|c|c|c|c|c|}
\hline & & \multicolumn{5}{|c|}{$\delta$} \\
\hline & & 1 & $1 / 2$ & $1 / 4$ & $1 / 6$ & $1 / 12$ \\
\hline \multirow[t]{2}{*}{$c=0$} & $\bar{L}_{S}(\delta)$ & 4.8 & 5.2 & 4.6 & 5.0 & 4.8 \\
\hline & $\bar{L}_{F}^{*}(\delta)$ & 4.8 & 5.2 & 4.6 & 5.0 & 4.8 \\
\hline \multirow[t]{2}{*}{$c=2.5$} & $\bar{L}_{S}(\delta)$ & 6.7 & 9.5 & 13.2 & 17.9 & 29.7 \\
\hline & $\bar{L}_{F}^{*}(\delta)$ & 6.7 & 7.3 & 6.8 & 7.0 & 6.9 \\
\hline \multirow[t]{2}{*}{$c=5$} & $\bar{L}_{S}(\delta)$ & 12.9 & 22.3 & 35.1 & 47.0 & 66.8 \\
\hline & $\bar{L}_{F}^{*}(\delta)$ & 12.8 & 13.9 & 13.2 & 13.5 & 13.2 \\
\hline \multirow[t]{2}{*}{$c=10$} & $\bar{L}_{S}(\delta)$ & 34.2 & 55.0 & 73.4 & 83.3 & 94.0 \\
\hline & $\bar{L}_{F}^{*}(\delta)$ & 34.2 & 35.5 & 35.2 & 36.6 & 36.5 \\
\hline \multirow[t]{2}{*}{$c=25$} & $\bar{L}_{S}(\delta)$ & 79.6 & 93.0 & 98.5 & 99.4 & 99.9 \\
\hline & $\bar{L}_{F}^{*}(\delta)$ & 80.1 & 82.7 & 83.5 & 84.1 & 84.5 \\
\hline
\end{tabular}


Table 2a: Upper tail fractiles from the asymptotic null distributions of the $L_{S}$ and $L_{F}^{*}$ statistics: constant level case.

\begin{tabular}{|c|c|c|c|c|c|c|c|}
\hline & & & \multicolumn{5}{|c|}{ Sampling Interval $\delta$} \\
\hline & & & 1 & $1 / 2$ & $1 / 4$ & $1 / 6$ & $1 / 12$ \\
\hline \multirow{9}{*}{$L_{S}$} & \multirow{3}{*}{$\lambda=0.25$} & 0.90 & 0.347 & 0.196 & 0.124 & 0.101 & 0.080 \\
\hline & & 0.95 & 0.461 & 0.259 & 0.164 & 0.134 & 0.107 \\
\hline & & 0.99 & 0.743 & 0.416 & 0.264 & 0.217 & 0.176 \\
\hline & \multirow{3}{*}{$\lambda=0.50$} & 0.90 & 0.347 & 0.260 & 0.221 & 0.209 & 0.197 \\
\hline & & 0.95 & 0.461 & 0.345 & 0.295 & 0.280 & 0.264 \\
\hline & & 0.99 & 0.743 & 0.562 & 0.490 & 0.468 & 0.448 \\
\hline & \multirow{3}{*}{$\lambda=0.75$} & 0.90 & 0.347 & 0.324 & 0.315 & 0.311 & 0.308 \\
\hline & & 0.95 & 0.461 & 0.434 & 0.420 & 0.416 & 0.412 \\
\hline & & 0.99 & 0.743 & 0.707 & 0.690 & 0.684 & 0.678 \\
\hline \multirow{9}{*}{$L_{F}^{*}$} & \multirow{3}{*}{$\lambda=0.25$} & 0.90 & 0.347 & 0.135 & 0.066 & 0.049 & 0.034 \\
\hline & & 0.95 & 0.461 & 0.180 & 0.088 & 0.064 & 0.045 \\
\hline & & 0.99 & 0.743 & 0.288 & 0.143 & 0.106 & 0.073 \\
\hline & \multirow{3}{*}{$\lambda=0.50$} & 0.90 & 0.347 & 0.194 & 0.134 & 0.117 & 0.102 \\
\hline & & 0.95 & 0.461 & 0.253 & 0.176 & 0.154 & 0.133 \\
\hline & & 0.99 & 0.743 & 0.416 & 0.287 & 0.249 & 0.213 \\
\hline & \multirow{3}{*}{$\lambda=0.75$} & 0.90 & 0.347 & 0.267 & 0.231 & 0.219 & 0.208 \\
\hline & & 0.95 & 0.461 & 0.354 & 0.307 & 0.293 & 0.278 \\
\hline & & 0.99 & 0.743 & 0.566 & 0.486 & 0.460 & 0.438 \\
\hline
\end{tabular}


Table 2b: Upper tail fractiles from the asymptotic null distributions of the $L_{S}$ and $L_{F}^{*}$ statistics: linear trend case.

\begin{tabular}{|c|c|c|c|c|c|c|c|}
\hline & & & \multicolumn{5}{|c|}{ Sampling Interval $\delta$} \\
\hline & & & 1 & $1 / 2$ & $1 / 4$ & $1 / 6$ & $1 / 12$ \\
\hline \multirow{9}{*}{$L_{S}$} & \multirow{3}{*}{$\lambda=0.25$} & 0.90 & 0.119 & 0.074 & 0.048 & 0.038 & 0.025 \\
\hline & & 0.95 & 0.149 & 0.092 & 0.060 & 0.047 & 0.031 \\
\hline & & 0.99 & 0.218 & 0.138 & 0.091 & 0.070 & 0.045 \\
\hline & \multirow{3}{*}{$\lambda=0.50$} & 0.90 & 0.119 & 0.085 & 0.065 & 0.057 & 0.050 \\
\hline & & 0.95 & 0.149 & 0.105 & 0.079 & 0.071 & 0.062 \\
\hline & & 0.99 & 0.218 & 0.152 & 0.115 & 0.104 & 0.094 \\
\hline & \multirow{3}{*}{$\lambda=0.75$} & 0.90 & 0.119 & 0.104 & 0.097 & 0.094 & 0.092 \\
\hline & & 0.95 & 0.149 & 0.130 & 0.121 & 0.118 & 0.116 \\
\hline & & 0.99 & 0.218 & 0.190 & 0.179 & 0.176 & 0.173 \\
\hline \multirow{9}{*}{$L_{F}^{*}$} & \multirow{3}{*}{$\lambda=0.25$} & 0.90 & 0.119 & 0.046 & 0.023 & 0.017 & 0.012 \\
\hline & & 0.95 & 0.149 & 0.057 & 0.028 & 0.021 & 0.014 \\
\hline & & 0.99 & 0.218 & 0.085 & 0.041 & 0.030 & 0.021 \\
\hline & \multirow{3}{*}{$\lambda=0.50$} & 0.90 & 0.119 & 0.067 & 0.046 & 0.040 & 0.035 \\
\hline & & 0.95 & 0.149 & 0.083 & 0.058 & 0.050 & 0.043 \\
\hline & & 0.99 & 0.218 & 0.121 & 0.065 & 0.074 & 0.063 \\
\hline & \multirow{3}{*}{$\lambda=0.75$} & 0.90 & 0.119 & 0.092 & 0.079 & 0.075 & 0.071 \\
\hline & & 0.95 & 0.149 & 0.114 & 0.098 & 0.093 & 0.088 \\
\hline & & 0.99 & 0.218 & 0.166 & 0.143 & 0.137 & 0.129 \\
\hline
\end{tabular}


Table 3a: Simulated rejection probabilities $(\times 100)$ for the mixed frequency DGP: $\lambda=0.25$.

\begin{tabular}{|c|c|c|c|c|c|c|c|c|c|c|c|}
\hline & \multirow[b]{2}{*}{$\delta$} & \multicolumn{5}{|c|}{ constant level } & \multicolumn{5}{|c|}{ linear trend } \\
\hline & & 1 & $1 / 2$ & $1 / 4$ & $1 / 6$ & $1 / 12$ & 1 & $1 / 2$ & $1 / 4$ & $1 / 6$ & $1 / 12$ \\
\hline \multirow{6}{*}{$c=0$} & $L_{S}$ & 5.3 & 4.9 & 4.5 & 4.1 & 4.7 & 4.7 & 5.4 & 5.8 & 5.2 & 5.0 \\
\hline & $\widehat{L}_{S}$ & 5.2 & 5.0 & 4.8 & 5.1 & 4.4 & 5.3 & 5.3 & 5.7 & 5.1 & 4.9 \\
\hline & $L_{S, 0}$ & 5.3 & 4.9 & 4.9 & 5.1 & 5.0 & 4.7 & 4.9 & 5.3 & 5.1 & 5.2 \\
\hline & $L_{F}^{*}$ & 5.3 & 5.1 & 4.6 & 4.8 & 4.9 & 4.7 & 5.4 & 5.4 & 4.7 & 4.9 \\
\hline & $\widehat{L}_{F}$ & 5.2 & 5.0 & 5.1 & 4.8 & 4.4 & 5.3 & 5.5 & 5.6 & 5.2 & 4.8 \\
\hline & $L_{F, 0}^{*}$ & 5.3 & 5.1 & 4.6 & 4.8 & 4.9 & 4.7 & 4.9 & 5.0 & 4.8 & 4.6 \\
\hline \multirow{6}{*}{$c=2.5$} & $L_{S}$ & 13.3 & 13.1 & 14.9 & 16.6 & 22.7 & 6.9 & 8.3 & 9.3 & 9.4 & 10.4 \\
\hline & $\widehat{L}_{S}$ & 8.6 & 8.7 & 8.9 & 9.6 & 10.4 & 6.2 & 6.3 & 7.0 & 6.3 & 6.6 \\
\hline & $L_{S, 0}$ & 13.3 & 10.3 & 10.3 & 11.0 & 13.1 & 6.9 & 6.6 & 6.7 & 6.4 & 6.8 \\
\hline & $L_{F}^{*}$ & 13.3 & 16.8 & 25.8 & 34.9 & 56.5 & 6.9 & 8.9 & 12.2 & 15.2 & 31.8 \\
\hline & $\widehat{L}_{F}$ & 8.6 & 9.3 & 13.6 & 18.0 & 38.1 & 6.2 & 6.7 & 7.7 & 8.7 & 16.2 \\
\hline & $L_{F, 0}^{*}$ & 13.3 & 16.8 & 25.0 & 33.3 & 52.2 & 6.9 & 8.4 & 11.2 & 14.5 & 27.4 \\
\hline \multirow{6}{*}{$c=5.0$} & $L_{S}$ & 30.7 & 32.2 & 37.2 & 41.1 & 49.2 & 13.2 & 16.5 & 20.3 & 21.1 & 25.9 \\
\hline & $\widehat{L}_{S}$ & 18.8 & 20.1 & 22.8 & 24.0 & 30.0 & 9.1 & 9.5 & 10.3 & 10.4 & 11.9 \\
\hline & $L_{S, 0}$ & 30.7 & 25.2 & 24.6 & 25.8 & 30.9 & 13.2 & 11.7 & 11.4 & 11.0 & 12.8 \\
\hline & $L_{F}^{*}$ & 30.7 & 39.2 & 52.9 & 64.1 & 81.6 & 13.2 & 18.8 & 29.7 & 39.8 & 68.1 \\
\hline & $\widehat{L}_{F}$ & 18.8 & 22.3 & 35.3 & 46.0 & 66.0 & 9.1 & 10.4 & 14.2 & 19.4 & 39.6 \\
\hline & $L_{F, 0}^{*}$ & 30.7 & 38.7 & 50.9 & 59.9 & 74.5 & 13.2 & 17.7 & 27.4 & 36.0 & 57.0 \\
\hline \multirow{6}{*}{$c=10$} & $L_{S}$ & 59.5 & 62.5 & 67.8 & 70.5 & 75.6 & 34.6 & 41.0 & 48.0 & 51.2 & 60.1 \\
\hline & $\widehat{L}_{S}$ & 45.7 & 48.7 & 54.9 & 58.3 & 66.2 & 20.8 & 22.4 & 24.9 & 26.9 & 33.0 \\
\hline & $L_{S, 0}$ & 59.5 & 52.2 & 51.0 & 51.8 & 56.7 & 34.6 & 28.1 & 27.5 & 27.6 & 31.8 \\
\hline & $L_{F}^{*}$ & 59.5 & 67.9 & 79.3 & 86.6 & 94.9 & 34.6 & 46.8 & 63.8 & 74.6 & 92.9 \\
\hline & $\widehat{L}_{F}$ & 45.7 & 55.1 & 70.0 & 77.2 & 85.7 & 20.8 & 25.1 & 37.7 & 49.6 & 70.1 \\
\hline & $L_{F, 0}^{*}$ & 59.5 & 66.5 & 74.9 & 79.9 & 85.6 & 34.6 & 43.8 & 58.2 & 66.9 & 79.6 \\
\hline
\end{tabular}


Table 3b: Simulated rejection probabilities $(\times 100)$ for the mixed frequency DGP: $\lambda=0.5$.

\begin{tabular}{|c|c|c|c|c|c|c|c|c|c|c|c|}
\hline & \multirow[b]{2}{*}{$\delta$} & \multicolumn{5}{|c|}{ constant level } & \multicolumn{5}{|c|}{ linear trend } \\
\hline & & 1 & $1 / 2$ & $1 / 4$ & $1 / 6$ & $1 / 12$ & 1 & $1 / 2$ & $1 / 4$ & $1 / 6$ & $1 / 12$ \\
\hline \multirow{6}{*}{$c=0$} & $L_{S}$ & 5.3 & 5.4 & 5.1 & 5.1 & 5.4 & 4.7 & 5.3 & 5.4 & 5.3 & 5.8 \\
\hline & $\widehat{L}_{S}$ & 4.9 & 5.1 & 4.9 & 4.9 & 4.8 & 5.4 & 5.3 & 5.5 & 5.1 & 5.2 \\
\hline & $L_{S, 0}$ & 5.3 & 4.8 & 4.9 & 5.0 & 5.0 & 4.7 & 5.1 & 4.9 & 5.1 & 5.1 \\
\hline & $L_{F}^{*}$ & 5.3 & 5.8 & 5.1 & 5.3 & 5.0 & 4.7 & 5.5 & 4.9 & 4.9 & 5.0 \\
\hline & $\widehat{L}_{F}$ & 4.9 & 5.5 & 4.6 & 4.6 & 4.9 & 5.4 & 5.4 & 5.7 & 5.0 & 4.9 \\
\hline & $L_{F, 0}^{*}$ & 5.3 & 5.4 & 4.9 & 5.2 & 4.9 & 4.7 & 5.4 & 4.9 & 4.7 & 4.8 \\
\hline \multirow{6}{*}{$c=2.5$} & $L_{S}$ & 13.3 & 16.4 & 23.8 & 29.5 & 42.1 & 6.9 & 8.4 & 9.7 & 11.0 & 16.5 \\
\hline & $\widehat{L}_{S}$ & 7.4 & 9.1 & 11.2 & 13.7 & 21.3 & 5.8 & 6.5 & 7.1 & 7.5 & 9.5 \\
\hline & $L_{S, 0}$ & 13.3 & 12.9 & 16.7 & 19.6 & 26.8 & 6.9 & 7.4 & 7.9 & 8.6 & 11.6 \\
\hline & $L_{F}^{*}$ & 13.3 & 22.3 & 39.7 & 53.9 & 77.8 & 6.9 & 10.2 & 18.3 & 29.1 & 59.7 \\
\hline & $\widehat{L}_{F}$ & 7.4 & 12.2 & 25.6 & 40.1 & 65.9 & 5.8 & 7.0 & 11.5 & 18.3 & 44.2 \\
\hline & $L_{F, 0}^{*}$ & 13.3 & 21.4 & 38.4 & 52.3 & 74.5 & 6.9 & 10.0 & 17.8 & 27.7 & 55.8 \\
\hline \multirow{6}{*}{$c=5.0$} & $L_{S}$ & 30.7 & 38.1 & 49.7 & 56.3 & 68.1 & 13.2 & 18.1 & 22.6 & 27.6 & 41.1 \\
\hline & $\widehat{L}_{S}$ & 15.8 & 21.1 & 29.5 & 36.4 & 48.8 & 8.0 & 9.8 & 13.0 & 14.9 & 23.0 \\
\hline & $L_{S, 0}$ & 30.7 & 31.9 & 38.4 & 43.9 & 54.2 & 13.2 & 14.5 & 17.0 & 20.3 & 29.7 \\
\hline & $L_{F}^{*}$ & 30.7 & 47.4 & 69.0 & 80.2 & 93.2 & 13.2 & 23.6 & 45.8 & 64.1 & 88.9 \\
\hline & $\widehat{L}_{F}$ & 15.8 & 30.5 & 54.5 & 68.5 & 84.8 & 8.0 & 12.6 & 28.8 & 45.5 & 74.9 \\
\hline & $L_{F, 0}^{*}$ & 30.7 & 46.3 & 66.6 & 77.4 & 89.7 & 13.2 & 22.9 & 44.2 & 60.5 & 84.0 \\
\hline \multirow{6}{*}{$c=10$} & $L_{S}$ & 59.5 & 66.9 & 74.6 & 79.2 & 86.4 & 34.6 & 44.0 & 55.4 & 62.1 & 75.0 \\
\hline & $\widehat{L}_{S}$ & 43.0 & 52.7 & 63.7 & 69.2 & 78.7 & 17.3 & 23.2 & 33.3 & 41.1 & 55.4 \\
\hline & $L_{S, 0}$ & 59.5 & 60.2 & 66.4 & 70.8 & 78.4 & 34.6 & 36.6 & 43.9 & 49.4 & 63.1 \\
\hline & $L_{F}^{*}$ & 59.5 & 74.7 & 89.1 & 94.2 & 98.2 & 34.6 & 55.9 & 79.3 & 90.7 & 98.1 \\
\hline & $\widehat{L}_{F}$ & 43.0 & 64.2 & 82.6 & 88.5 & 93.9 & 17.3 & 33.3 & 62.5 & 77.9 & 90.8 \\
\hline & $L_{F, 0}^{*}$ & 59.5 & 73.2 & 86.7 & 91.5 & 95.1 & 34.6 & 54.3 & 76.0 & 86.6 & 94.8 \\
\hline
\end{tabular}


Table 3c: Simulated rejection probabilities $(\times 100)$ for the mixed frequency DGP: $\lambda=0.75$.

\begin{tabular}{|c|c|c|c|c|c|c|c|c|c|c|c|}
\hline & \multirow[b]{2}{*}{$\delta$} & \multicolumn{5}{|c|}{ constant level } & \multicolumn{5}{|c|}{ linear trend } \\
\hline & & 1 & $1 / 2$ & $1 / 4$ & $1 / 6$ & $1 / 12$ & 1 & $1 / 2$ & $1 / 4$ & $1 / 6$ & $1 / 12$ \\
\hline \multirow{6}{*}{$c=0$} & $L_{S}$ & 5.3 & 4.9 & 4.9 & 4.8 & 4.5 & 4.7 & 5.1 & 5.1 & 5.5 & 4.7 \\
\hline & $\widehat{L}_{S}$ & 5.1 & 4.9 & 5.0 & 5.3 & 4.5 & 5.2 & 5.4 & 4.9 & 5.5 & 5.1 \\
\hline & $L_{S, 0}$ & 5.3 & 5.3 & 4.7 & 4.9 & 4.9 & 4.7 & 5.1 & 4.7 & 5.3 & 5.0 \\
\hline & $L_{F}^{*}$ & 5.3 & 4.9 & 4.5 & 4.7 & 4.4 & 4.7 & 5.1 & 4.6 & 4.5 & 4.9 \\
\hline & $\widehat{L}_{F}$ & 5.1 & 5.4 & 5.0 & 5.1 & 4.6 & 5.2 & 5.6 & 5.1 & 5.6 & 4.9 \\
\hline & $L_{F, 0}^{*}$ & 5.3 & 5.0 & 4.7 & 5.0 & 4.5 & 4.7 & 5.1 & 4.5 & 4.4 & 4.8 \\
\hline \multirow{6}{*}{$c=2.5$} & $L_{S}$ & 13.3 & 18.9 & 28.9 & 35.0 & 51.0 & 6.9 & 8.9 & 11.7 & 15.7 & 23.8 \\
\hline & $\widehat{L}_{S}$ & 8.7 & 11.0 & 17.5 & 22.9 & 34.4 & 5.9 & 7.3 & 8.6 & 10.6 & 15.5 \\
\hline & $L_{S, 0}$ & 13.3 & 16.3 & 23.2 & 28.7 & 41.0 & 6.9 & 8.1 & 9.5 & 12.7 & 19.2 \\
\hline & $L_{F}^{*}$ & 13.3 & 25.8 & 49.6 & 65.6 & 86.8 & 6.9 & 11.8 & 26.1 & 42.5 & 76.9 \\
\hline & $\widehat{L}_{F}$ & 8.7 & 17.6 & 40.9 & 57.4 & 80.9 & 5.9 & 8.9 & 19.5 & 32.2 & 67.4 \\
\hline & $L_{F, 0}^{*}$ & 13.3 & 25.8 & 49.8 & 65.5 & 85.8 & 6.9 & 11.7 & 25.5 & 41.5 & 75.1 \\
\hline \multirow{6}{*}{$c=5.0$} & $L_{S}$ & 30.7 & 42.6 & 56.8 & 64.2 & 76.2 & 13.2 & 19.8 & 29.7 & 38.5 & 54.8 \\
\hline & $\widehat{L}_{S}$ & 18.9 & 28.4 & 41.5 & 49.9 & 63.5 & 8.6 & 12.7 & 19.5 & 25.3 & 40.2 \\
\hline & $L_{S, 0}$ & 30.7 & 38.5 & 49.5 & 57.0 & 69.9 & 13.2 & 17.4 & 25.0 & 31.6 & 47.5 \\
\hline & $L_{F}^{*}$ & 30.7 & 53.0 & 77.1 & 87.3 & 96.5 & 13.2 & 29.1 & 59.8 & 77.6 & 95.1 \\
\hline & $\widehat{L}_{F}$ & 18.9 & 42.2 & 69.0 & 81.3 & 92.5 & 8.6 & 20.2 & 48.1 & 66.7 & 89.9 \\
\hline & $L_{F, 0}^{*}$ & 30.7 & 52.8 & 76.6 & 86.6 & 95.4 & 13.2 & 29.2 & 58.9 & 76.3 & 93.7 \\
\hline \multirow{6}{*}{$c=10$} & $L_{S}$ & 59.5 & 70.6 & 81.2 & 86.0 & 91.8 & 34.6 & 47.7 & 63.8 & 72.0 & 85.1 \\
\hline & $\widehat{L}_{S}$ & 44.6 & 58.1 & 71.2 & 77.3 & 85.7 & 20.1 & 32.9 & 49.0 & 58.1 & 74.3 \\
\hline & $L_{S, 0}$ & 59.5 & 67.2 & 76.1 & 81.4 & 88.9 & 34.6 & 44.2 & 58.8 & 66.7 & 80.2 \\
\hline & $L_{F}^{*}$ & 59.5 & 79.4 & 93.1 & 96.5 & 98.9 & 34.6 & 63.6 & 89.1 & 95.5 & 99.2 \\
\hline & $\widehat{L}_{F}$ & 44.6 & 70.9 & 88.6 & 93.4 & 96.5 & 20.1 & 49.0 & 80.4 & 90.1 & 96.6 \\
\hline & $L_{F, 0}^{*}$ & 59.5 & 78.9 & 92.5 & 95.8 & 98.2 & 34.6 & 63.0 & 87.9 & 94.3 & 98.4 \\
\hline
\end{tabular}




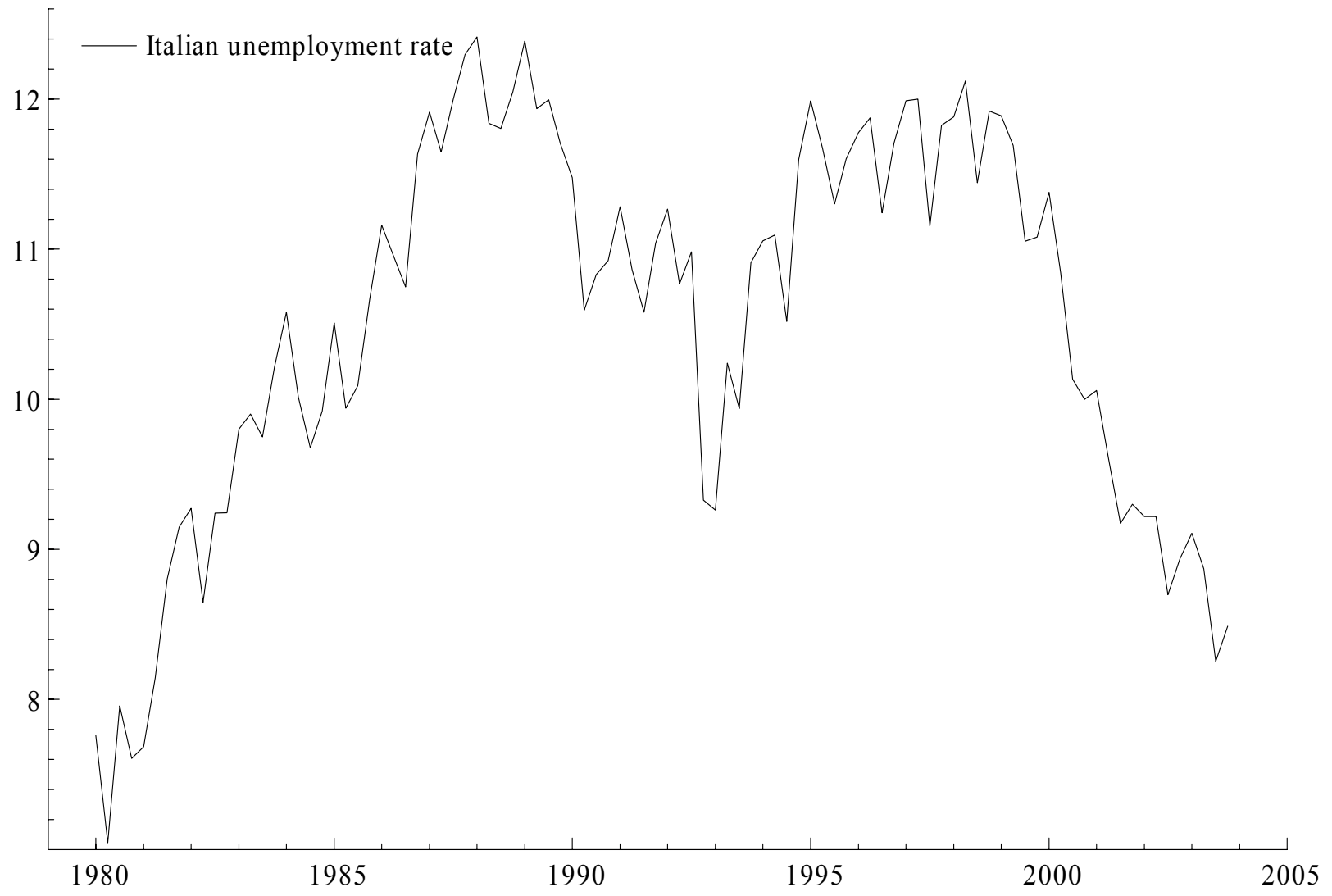

Figure 1. Italian unemployment rate, 1980Q1-2003Q4. 


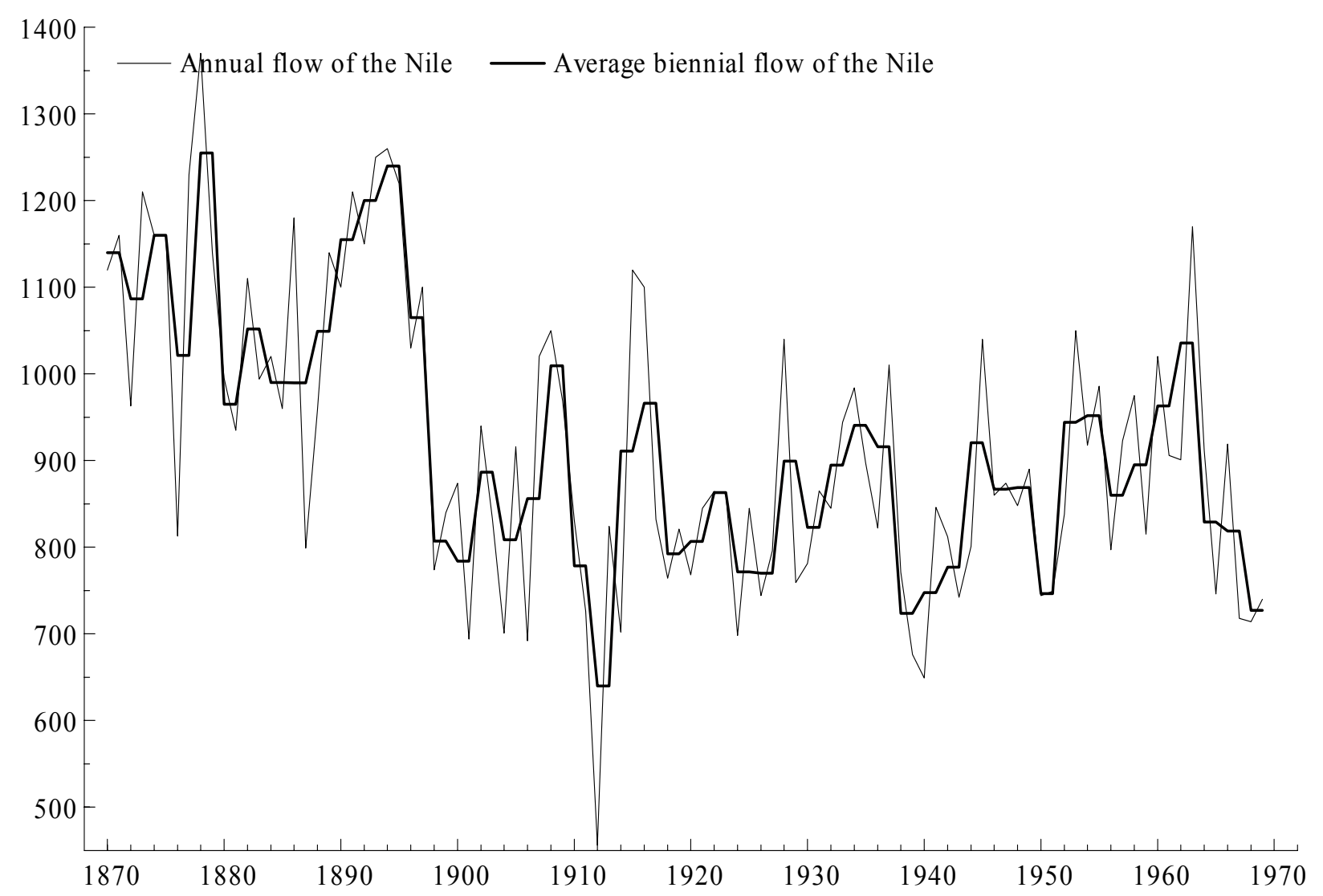

Figure 2. Flow of the Nile, 1871-1970. 


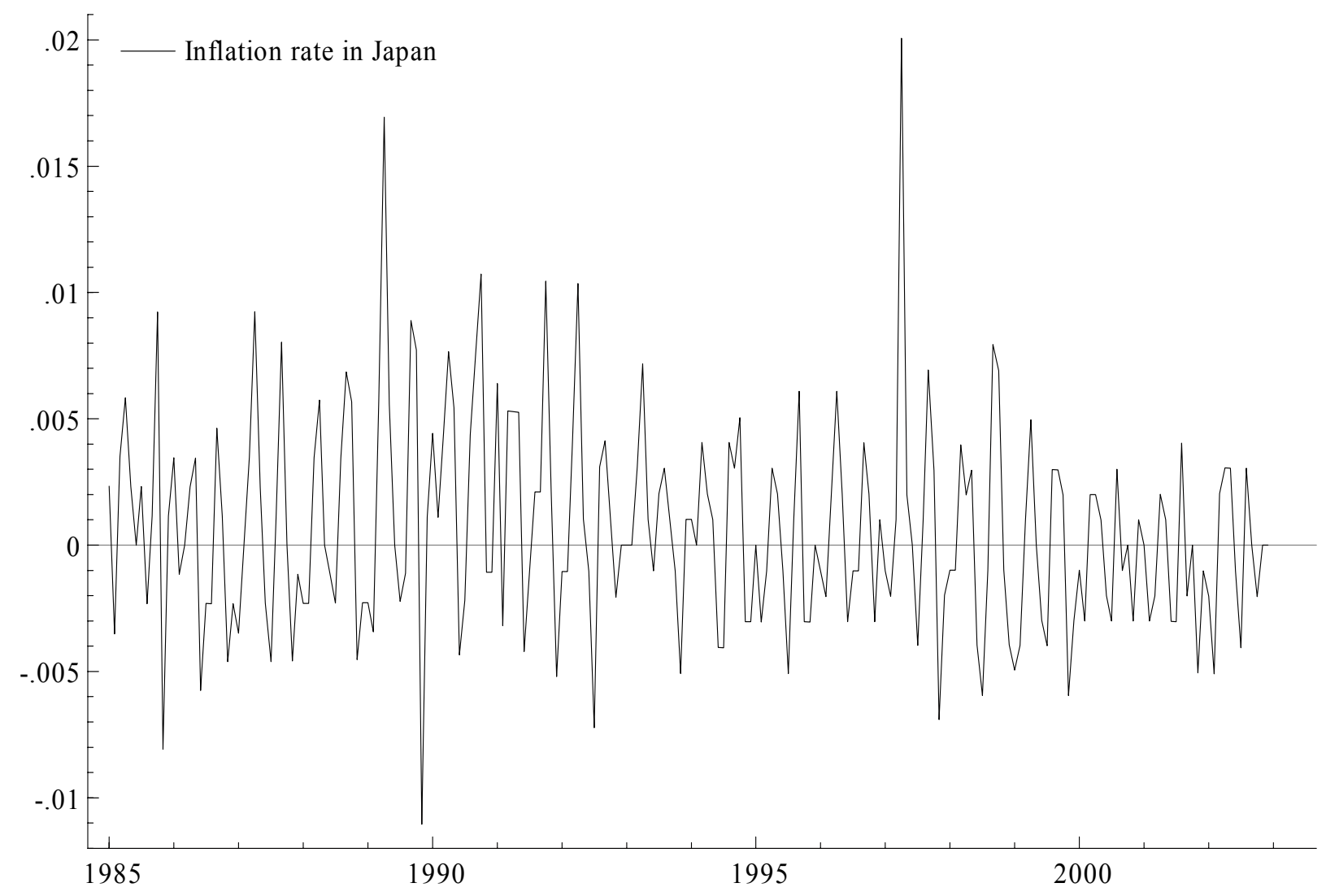

Figure 3. Inflation rate in Japan, 1985M1-2002M12. 